\title{
3. GIFTGAS, POCKEN UND TYPHUS ABDELKRIMS SUCHE NACH HUMANITÄRER HILFE
}

\subsection{Die Kriegsgreuel}

Abdelkrim wollte innerhalb seiner »Rif-Republik« wie auch nach außen mit Blick auf Europa >Progressivität $\iota$ und \Modernität demonstrieren, insbesondere was den technischen Fortschritt anbetraf ${ }^{1}$. Seine Erfahrungen als Mitglied der spanischen Kolonialverwaltung hatten in ihm die Vorstellung reifen lassen, daß die internen Strukturen des Rifs durch Adaptionen aus der ımoderneren< Welt verbessert werden sollten. Europa war Abdelkrims Vorbild ${ }^{2}$. Aber ihm lag auch viel daran zu beweisen, daß die Rifbevölkerung selbst in der Lage war, einen eigenen Staat zu verwalten. Für die vorgesehene Modernisierung benötigte Abdelkrim ausländische Helfer, Experten und Fachleute - Ärzte, Ingenieure, Techniker, Mechaniker, politische Berater, militärische Instrukteure. Er benötigte europäische Unterstützer im humanitären, militärischen, technischen, wirtschaftlichen und politischen Bereich. Mit ihnen vollführte Abdelkrim einen Spagat zwischen Traditionalität und Modernität ${ }^{3}$. Traditionalität bedeutete im wesentlichen die Bewahrung der Unabhängigkeit im Einklang mit gewohnten Lebensweisen und mit dem Islam. Modernität bedeutete den Versuch, mit europäischer Technologie sowie mit technischen und militärischen Instrukteuren - zumeist Deserteure aus den feindlichen Fremdenlegionen - im Kampf gegen die französisch-spanischen Protektoratstruppen bestehen zu können, durch Kontakte zu europäischen Wirtschaftsleuten die Infrastruktur innerhalb des Rifs zu verbessern und mit Hilfe politischer Berater die internationale Anerkennung des Rifstaates herbeizuführen.

Genauso benötigte Abdelkrim für die medizinische Versorgung von Kämpfern und Zivilisten humanitäre Helfer. Der Krieg traf im besonderen Maße die Zivilbevölkerung im Rif. Da die traditionellen Heilmethoden der Rifkabylen nicht ausreichten, wandte sich Abdelkrim vor allem an das europäische Ausland mit der Bitte um Hilfe.

' Abderrahim HAROUCHI, Les États-Unis face à la République du Rif, in: Abd el-Krim et la République du Rif, S. 367-373, S. 368; FlemInG, Primo de Rivera and Abd-el-Krim, S. 225; DAOUD, Abdelkrim, S. 152.

${ }^{2}$ LA PORTE, La atracción del imán, S. 130f;; AYACHe, La Guerte du Rif, S. 249; SHINAR, Abd al-Qadir, S. 173.

${ }^{3}$ Vgl. Mohammed Tahtahs Dissertationstitel »Entre pragmatisme, réformisme et modernisme« (1995) oder Maria-Rosa de Madariagas Kapitel "Entre stradición` y ımodernidad«« [MADARIAGA, España y el Rif, S. 552-565]. 
Die Kampfhandlungen forderten unzählige Tote, Verwundete und Vermißte auf allen Seiten. Offizielle Angaben über die Verlustzahlen wirken geschönt. Hiernach wurden etwa in der spanischen Armee zwischen 1921 und 1926 17020 Tote und Vermißte sowie 23610 Verwundete gezählt ${ }^{4}$ - angesichts der verheerenden Niederlagen der Spanier in Annual (1921) und während des Rückzugs aus Chichaouen (1924) wohl Untertreibungen, denn allein die spanische Fremdenlegion registrierte während des Rifkrieges schon 8381 Tote, Vermißte und Verwundete ${ }^{5}$. Nicht anders verhält es sich mit Angaben über französische Verluste. Anthony Clayton etwa gibt mit Bezug auf französische Quellen für die Zeit zwischen April 1925 und Mai 1926 die Zahl von 2162 Toten und Vermißten an ${ }^{6}$. Hingegen zählten die Franzosen Arnold Toynbee zufolge bereits bei den Kämpfen bis Mitte Oktober 19252176 Tote und Vermißte. Hinzu kamen 8297 Verwundete ${ }^{7}$. Vielfach wurden sowohl von der französischen als auch von der spanischen Armee die indigenen Hilfstruppen nicht in die Verluststatistiken aufgenommen. Die Dunkelziffer war daher sehr hoch. Niemand weiß, wie viele Rifkämpfer oder Zivilisten während des Krieges ums Leben kamen oder verwundet wurden. Schätzungsweise gehen die Zahlen weit in die Tausende ${ }^{8}$.

Jegliche Romantisierung der Kämpfe verbietet sich von selbst. Alle Seiten begingen unglaubliche und haarsträubende Grausamkeiten. Schlimme Exzesse waren an der Tagesordnung. Zwei Massaker an Spaniern in Annual und Jebel Aroui bildeten 1921 den Anfang des Rifkrieges. Als die spanischen Truppen zwischen September und November 1921 zu den Stätten der Niederlagen zurückkehrten, bot sich ihnen ein Bild des Schreckens. Leichen - allein in Jebel Aroui mindestens 800 - lagen in der Sonne, zum großen Teil verstümmelt, ohne Köpfe, zweigeteilt, arm- und beinlos, ohne Zungen, Augen oder Genitalien'. Rituelle Verstümmelungen von Feinden waren in Nordmarokko durchaus übliche Praktiken von Rache bei intra- oder intertribalen Blutfehden, die

\footnotetext{
${ }^{4}$ Fleming, Primo de Rivera and Abd-el-Krim, App. A, S. 398 mit Bezug auf Hernandez HerRera, GARCIA Figueras, Acción de España, Bd. 1, S. 683.

${ }^{5}$ ÁlvareZ, The Betrothed of Death 2001, S. 206, S. 235.

${ }^{6}$ Clayton, France, S. 110. Vgl. Nos pertes au Maroc, in: Écho de Paris, 30.5.1926.

${ }^{7}$ TOYNBEE, The Islamic World, S. 149. Vgl. HARRIS, France, S. 246f. Die amerikanische Zeitschrift „Current History Magazine" ging von 11412 Toten und Verwundeten auf der französischen Seite aus [The End of the War in Morocco, in: The Current History Magazine 24 (1926) S. 551-553, S. 553].

${ }^{8}$ KUNZ, MÜller, Giftgas, S. 81; Álvarez, The Betrothed of Death 2001, S. 62, Anm. 11.

${ }^{9}$ WoOlman, Rebels in the Rif, S. 103; VIllaloBOS, El sueño colonial, S. 133-135; ÁlvaREZ, The Betrothed of Death 2001, S. 51f., S. 54, S. 72, Anm. 47. Beispiele für Greueltaten in: HaRRIS, France, S. 72; SEHRING, Auf den Schlachtfeldern, S. 48; SABLOTNY, Légionnaire, S. 164; Ex-Légionnaire 1384 of the Légion Etrangère, Hell Hounds of France, London 1932, S. 8; Alfred LORENSEN, Mit der Legion in Syrien und Marokko, in: Süddeutsche Monatshefte 23 (1925) S. 416-418, S. 418; WARD PRICE, Extra-Special Correspondent, S. 166; WOOLMAN, Rebels in the Rif, S. 201.
} 
nun auf den Kampf gegen die zudem christlichen Gegner übertragen wurden ${ }^{10}$. Abdelkrim dementierte zunächst Greueltaten und behauptete, entsprechende Fotos seien gefälscht ${ }^{11}$. Nach dem Krieg rechtfertigte er sich, er habe die Gewalttaten limitieren können: "Wenn ich nicht die allerstrengsten Befehle erteilt und unzählige Male mit Todesstrafe gedroht hätte, um dem Schlachten Einhalt zu tun, würden die Verluste unserer Feinde jedes vorstellbare Maß überschritten haben ${ }^{12}$.

Insbesondere die spanische Fremdenlegion stand den Rifkabylen in nichts nach. Dörfer, in denen es gar keine Rifkämpfer gab, wurden zerstört und niedergebrannt, Frauen und Kinder erschossen oder in die Flammen getrieben - wie im Sommer 1924 bei den Beni Saïd ${ }^{13}$. Erste Berichte an das britische Außenministerium, daß spanische Offiziere Fotos von geköpften Rifsoldaten vorgezeigt hätten, wurden im Januar 1922 nicht ernstgenommen ${ }^{14} .1924$ verdichteten sich die Hinweise, daß die Führung der spanischen Fremdenlegion ein Kopfgeld auf Rifkämpfer aussetzte ${ }^{15}$. Am 14. September 1925 erreichte die schauerliche Praxis ihren Höhepunkt. Während einer Parade in Tetuan defilierte eine Abteilung der spanischen Fremdenlegion an General Primo de Rivera vorbei. An den Bajonetten der Soldaten hingen Ohren und Zungen getöteter Rifkabylen. Unter dem makabren Titel »Totenkopfparade« berichtete auch die "Deutsche Allgemeine Zeitung" davon ${ }^{16} .1927 \mathrm{kam}$ es in Dörfern, die als Basis des Aufstandes galten, auch zu Massenerschießungen ${ }^{17}$.

Gegen Angriffe aus der Luft konnten sich die Rifkabylen nicht verteidigen. Seit Beginn des Krieges bombardierten spanische und später alliierte Flugzeuge bevorzugt zivile Ziele, wodurch der $\mathrm{Haß}$ der Rifkämpfer auf ihre Gegner noch verstärkt wurde ${ }^{18}$. »Mittelalterliche Grausamkeit« warf Abdelkrim den

${ }^{10}$ DRIESSEN, Images of Spanish Colonialism, S. 62; FURNEAUX, Abdel Krim, S. 79.

"Dies sagte Abdelkrim zum Briten John Arnall, der ihn im Rif besuchte [PRO-FO 371, 8354, 4882: John Arnall an Arthur Field, 29.5.1922].

${ }_{12}$ ABDELKRM, Memoiren, S. 72.

${ }^{13}$ PRO-FO 636, 7: Consul of Tetuan an CG Tangier, 25.9.1924.

${ }^{14}$ PRO-FO 371, 8341, 171, 101: John Amall an CG Tangier, 21.12.1921; PRO-FO 371, 8341, 171, 98: CG Tangier an FO, 4.1.1922.

${ }^{15}$ Otto HAENTSCHEL, In der Hölle von Tetuan. Mit den Spaniern gegen die Marokkaner, in: Vossische Zeitung, 2.5.1925; SABLOTNY, Légionnaire, S. 117, S. 124, S. 206; CADN Maroc DAI 192: Tabor de police $\mathrm{N}^{\circ} 1$, Bericht »Interrogatoire d'un déserteur de la Légion étrangère espagnole, de nationalité allemande«, 16.10.1925; PA-AA R71927: Preußischer Minister des Innern an AA, 19.6.1926; SEHRING, Auf den Schlachtfeldern, S. 33.

${ }^{16}$ Totenkopfparade, in: Deutsche Allgemeine Zeitung, 18.9.1925; PRO-FO 371, 11080, 39, 142: Consul of Tetuan an CG Tangier, 14.9.1925; CADN Tanger C37: EMA, $2^{c}$ bureau, Bericht "Atrocités espagnoles«, 17.9.1925; WOOLMAN, Rebels in the Rif, S. 201f.; VILLALOBOS, El sueño colonial, S. 148.

${ }^{17}$ KUNZ, wCon ayuda«, S. 184.

${ }^{18}$ RUE, Tribune, 23.6.1925; WOOLMAN, Rebels in the Rif, S. 202; PRO-FO 371, 8354, 4882: John Arnall an Arthur Field, 29.5.1922; Two Englishmen among the Riff Tribesmen. Secret Mission to Abdel Krim, in: Manchester Guardian, 13.12.1924. 
spanischen Truppen in einer Erklärung vom Mai 1922 vor, weil die Luftbombardements vor allem unbeteiligte Frauen und Kinder träfen ${ }^{19}$. Hunger setzten die spanischen wie auch die französischen Truppen als gezielte Waffe ein. Die Hungersnöte von Anfang $1923^{20}$, Ende $1924^{21}$ und Anfang 1926 resultierten aus den immer dichter wirkenden Blockademaßnahmen der Spanier und ab dem Sommer 1925 aus der alliierten Umklammerung des Rifs durch Spanien und Frankreich. Die Grenzen nach Algerien und Französisch-Marokko waren geschlossen, und die Nachschubversorgung war damit praktisch unterbunden. Märkte und Felder waren das Ziel von Luftangriffen ${ }^{22}$. Die Zahl von 11586 Lufteinsätzen, die allein französische Staffeln von Mai 1925 bis Mai 1926 gegen Ziele im Rif flogen - wobei eine Bombenlast in Höhe von 1434162 Kilogramm abgeworfen wurde -, verdeutlicht das für damalige Verhältnisse immense Ausmaß dieses Luftkrieges ${ }^{23}$.

Auf französischer Seite gab es sogar eine - offiziell in die Armee des Sultans integrierte - amerikanische Flugzeugstaffel, die nach der aus dem Ersten Weltkrieg bekannten Escadrille Lafayette benannte Escadrille américaine. Die amerikanischen Piloten brachten es zwischen Juli und November 1925 schon allein auf 350 Einsätze $e^{24}$. Nach der Anfang November 1925 erfolgten Bombardierung des völlig schutzlosen und zur offenen Stadt erklärten Chichaouen wurde die Staffel der Amerikaner aufgelöst - nicht, weil dort lediglich wehrlose Zivilisten umgekommen waren, sondern wegen der internationalen Proteste, daß die Maschinen nach dem Einsatz verbotenerweise in der neutralen Stadt Tanger gelandet waren ${ }^{25}$.

Der schlimmste Auswuchs des Luftkrieges war der Einsatz von Giftgas, den vor allem der von einem "genozidalen Rassismus« durchsetzte spanische König Alfonso XIII. mit dem Ziel, die eng mit Abdelkrim verbündeten Kabylen

${ }^{19}$ Walter B. HARRIS, Spain's Moroccan War. Moorish Leader's Challenge. Charges of Cruelty, in: Times, 30.5.1922; Une déclaration d'Abd el Krim, in: BCAF 32 (1922) S. 301f., S. 302.

${ }^{20}$ PA-AA R72042, L057456: Konsulat Tetuan an Botschaft Madrid, 2.2.1923.

${ }^{21}$ SABLOTNY, Légionnaire, S. 270.

${ }^{22}$ Fleming, Primo de Rivera and Abd-el-Krim, S. 324f.; PenNell, A Country, S. 210.

${ }^{23}$ Simone PESQUIÈS-COURBIER, L'aéronautique militaire française dans la Guetre du Rif, in: Revue du nord 285 (1990) S. 317-367, S. 344f., S. 349f., S. 353f.; KHARCHICH, Observations, S. 223-226; Alfred von MIERKA, Die militärische Lage in Marokko IV. Die EinschlieBung des Rifgebiets im Osten, in: Kölnische Zeitung, 23.10.1925; DERS., Der letzte Akt des marokkanischen Feldzugs. Die große spanisch-marokkanische Offensive, in: Kölnische Zeitung, 16.6.1926.

${ }^{24}$ Papers Relating to the Foreign Relations of the United States 1925, Bd. 2, S. 613 mit Bezug auf einen Artikel der »Temps«, 14.11.1925.

${ }^{25}$ TNLA, BNS, 1, Williams: Walter Harris an Harold Williams, 3.11.1925; PRO-FO 371, 11076, 18, 152: CG Tangier an FO, 3.11.1925; PRO-FO 371, 11076, 18, 154: FO an Embassy of Paris, 5.11.1925; VAIDON, Tangier, S. 198; HARRIS, France, S. 300. 
des Rifs auszulöschen, vorantrieb ${ }^{26}$. Der Rifkrieg war, nachdem Giftgas während des Ersten Weltkrieges lediglich vom Boden aus verwendet worden war, nicht nur »der erste >aerochemische` Krieg der Geschichte $«{ }^{27}$, sondern auch der erste militärische Konflikt im "Jahrhundert der Extreme ${ }^{28}$, in dem es gezielt gegen die Zivilbevölkerung eingesetzt wurde. Neben Spanien benutzten auch Großbritannien (Irak 1920) und Italien (Libyen 1923-1924, 1927-1928, Abessinien 1935-1936) in Kolonialkonflikten Giftgase ${ }^{29}$.

Direkt nach der Niederlage von Annual im Jahre 1921 knüpfte das spanische Militär Kontakte zur führenden Chemienation Deutschland ${ }^{30}$. Die Verhandlungen waren streng geheim, denn sie verstießen eklatant gegen den Versailler Vertrag. Dr. Hugo Stolzenberg, der Leiter der Firma Kampfstoffverwertung in Munsterlager-Breloh, versorgte Spanien bis 1923 mit 500 bis 600 Tonnen Phosgen, einem Lungengift, und Clark, einem Nasen- und Rachenreizstoff. Am 10. Juni 1922 wurde der Bau einer Chemiefabrik für die Herstellung von Giftgasen in La Marañosa bei Madrid beschlossen. Stolzenberg brachte die Entwicklung einer neuartigen Gasbombe für die Luftwaffe voran und entwarf eine Strategie für den Marokkokrieg, die vor allem den Einsatz des hochwirksamen und extrem gefährlichen Hautgiftes Lost (oder Yperit bzw. Senfgas) im Hinterland vorsah. Der Aufenthalt der Rifkabylen in Dörfern, das Abhalten

${ }^{26}$ BALFOUR, Deadly Embrace, S. 135.

${ }^{27}$ KUNZ, MŬLLER, Giftgas, S. 13. Die Studie der Militärhistoriker Rudibert Kunz und RolfDieter Müller ist für diese Thematik grundlegend, basiert aber auf rein deutschen Quellen. $\mathrm{Zu}$ den deutschen Gaslieferungen auch: Hubert BRIEDEN, Giftgasbomben für SpanischMarokko. Deutschlands Beteiligung am ersten »aero-chemischen« Krieg, in: Junge Welt, 17.7.1998; Dieter MARTINETZ, Vom Giffpfeil zum Chemiewaffenverbot. Zur Geschichte der chemischen Kampfmittel, Thun, Frankfurt a. M. 1995, S. 154-159; Volker ULRICH, Völkermord im Rif, in: Die Zeit, 1.2.1991. Sie wiederholen weitestgehend Kunz' und Müllers Ergebnisse bezïglich des Rifkrieges. Sebastian Balfours Kapitel "The Secret History of Chemical Warfare against Moroccans" in seinem Buch "Deadly Embrace» (2002) verbessert und ergänzt ihre Studie anhand spanischer Quellen [BALFour, Deadly Embrace, S. 123157].

${ }^{28}$ BRUMLIK, Micha, Das Jahrhundert der Extreme, in: WOJAK, MEINL (Hg.), Völkermord, S. $19-36$.

${ }^{29}$ BALFOUR, Deadly Embrace, S. 127f.; KunZ, 》Con ayuda«, S. 164f.; Angelo DeL BOCA, Faschismus und Kolonialismus. Der Mythos von den »anständigen Italienern«, in: WOJAK, MENL (Hg.), Völkermord, S. 193-202, S. 194f.; Aram MATTIOLl, Die vergessenen Kolonialverbrechen des faschistischen Italien in Libyen 1923-1933, in: WOJAK, MEINL (Hg.), Völkermord, S. 203-226, S. 212; DERS., Entgrenzte Kriegsgewalt. Der italienische Giftgaseinsatz in Abessinien 1935-1936, in: Vierteljahreshefte für Zeitgeschichte 51 (2003) S. 311337.

${ }^{30}$ KUNZ, MÜLlER, Giftgas, S. 20, S. 59, S. 62; BALFour, Deadly Embrace, S. 132f.; Hartmut STILLER, Gaskrieg, Völkerrecht, Geheimrüstung. Zur Frage der Beschränkung von bakteriologischen und chemischen Waffen in der Zwischenkriegszeit, in: Wissenschaft \& Frieden 12 (1995), H. 3, S. 40-44, S. 42; BRIEDEN, Giftgasbomben, 17.7.1998; MARTINETZ, Giftpfeil, S. 154f. Die deutschen Gaslieferungen an Spanien verschweigt Mimoun Charqi völlig [CHARQI, Abdelkrim, S. 153-158]. 
von Märkten und die Feldarbeit sollten durch die Bombardements unmöglich gemacht werden, um so Abdelkrim zur Aufgabe zu zwingen ${ }^{31}$. Geringe Mengen an Lost reichten wegen seiner extremen Haftbarkeit bereits aus, um ganze Landstriche, den Bewuchs, Nahrungsmittel oder Straßen hochgradig und langfristig $\mathrm{zu}$ verseuchen. Normale Bekleidung reichte als Schutz nicht aus. Schmerzhafte und schwer heilende Blasen und Wunden konnten tödlich sein. Der Verzehr vergifteter Nahrungsmittel oder Flüssigkeiten führte zu Zerstörungen und Geschwüren im Magen-Darm-Bereich und hatte fast immer den Tod zur Folge ${ }^{32}$.

Seit Oktober 1921 verschoß die spanische Artillerie bereits Granaten mit erstickenden Kampfstoffen ${ }^{33}$. Während der Schlacht von Tizi Azza, die am 15. Juli 1923 begann, setzte sie zum ersten Mal Senfgasgranaten ein ${ }^{34}$. Diese Schlacht - das "Verdun des Rifs $\aleph^{35}$ - wurde erstmals während des Rifkrieges durch Giftgaseinsätze entschieden ${ }^{36}$. Im Juni 1924 wurden die ersten Senfgasbomben aus der Luft abgeworfen ${ }^{37}$. In größerem Stil und mit großem Effekt verwendete die spanische Luftwaffe Gase nach dem Verlust von Chichaouen Ende $1924^{38}$. Bei ungünstigen Windverhältnissen trafen sie gelegentlich die eigenen Leute. Ohne Rücksicht wurden angreifende Truppen in die gerade verseuchten Gebiete getrieben ${ }^{39}$. Der Rückzug der Spanier hinter die Primode-Rivera-Linie gegen Ende 1924 erfolgte gerade deshalb, um im Rif selbst ungestört und ungehemmt Gasbomben einsetzen zu können ${ }^{40}$. Diese müssen unweigerlich auch die spanischen und später französischen Kriegsgefangenen getroffen haben - wohl mit ein Grund dafür, daß der Einsatz von Gasen in der spanischen Öffentlichkeit streng geheim gehalten wurde.

${ }^{31}$ KUNZ, MÜLLER, Giftgas, S. 68, S. 72, S. 76-80, S. 151f.; BALFOUR, Deadly Embrace, S. 132f.; VIÑAS, Franco, S. 91-95; MARTINETZ, Giftpfeil, S. 155f.; Olaf GROEHLER, Der lautlose Tod. Einsatz und Entwicklung deutscher Giftgase von 1914 bis 1945, Reinbek 1989, S. 64-68, S. 75.

${ }^{32}$ KUNZ, MÜLleR, Giftgas, S. 125f.; Carlos LÁZARo AVILA, La forja de la aeronáutica militar, Marruecos, in: CARRASCo GARCíA, MuÑoz Bolaños (Hg.), Las campañas, S. 164-193, S. 183-186.

${ }^{33}$ KUNZ, „Con ayuda«, S. 159.

${ }^{34}$ BALfour, Deadly Embrace, S. 138.

${ }^{35}$ PANDO, Historia secreta, S. 263.

${ }^{36}$ KUNZ, »Con ayuda«, S. $172 \mathrm{f}$.

${ }^{37}$ BALFOUR, Deadly Embrace, S. 140; VIÑas, Franco, S. 98-101.

${ }^{38}$ Auszug aus dem geheimen Bericht von Hauptmann Grauert und Leutnant Jeschonnek über die Reise nach Spanien von April bis Juni 1925, in: KUNZ, MŪLLER, Giftgas, S. 199-211, S. 207; FLEMING, Primo de Rivera and Abd-el-Krim, S. $141 \mathrm{f}$.

${ }^{39}$ BALFOUR, Deadly Embrace, S. 143-146; PRO-FO 371, 11077, 39, 40: Embassy of Madrid an FO, 20.5.1925 mit Bezug auf einen Bericht des Militärattachés W.W.T. Torr; KUNZ, MÜLLER, Giftgas, S. $91 \mathrm{f}$.

${ }^{40}$ KUNZ, wCon ayuda«, S. 174; PA-AA R72043, L057788: Konsul Tetuan an Botschaft Madrid, 20.12.1924. 
Primo de Rivera wollte die Rifkabylen mit Hilfe des von Hugo Stolzenberg entwickelten besonders haftbaren Zäh-Lost im Herzen ihres Landes bestrafen $^{41} .110$ Tonnen Lost in etwa 10000 Giftgasbomben - zur Verschleierung im internen Sprachgebrauch zwischen Madrid und Marokko »bombas especiales« bzw. »bombas $X_{\text {« genannt }}{ }^{42}$ - wurden während des Rifkrieges von spanischen Flugzeugen auf vornehmlich zivile Ziele abgeworfen. Es muß Tausende von Toten, Vergifteten und Verwundeten gegeben haben. Die genaue Zahl ist unbekannt. Nur wenige Nachrichten drangen hierüber aus dem Rif nach außen ${ }^{43}$.

Ende 1923 berichteten französische Behörden aus Oujda, daß es zahlreiche Krankheiten im Rif gebe ${ }^{44}$. Typhus brach im Sommer 1925 und Anfang 1926 aus $^{45}$. Rudibert Kunz und Rolf-Dieter Müller vermuten, daß diese Epidemien aufgrund der ähnlichen Folgesymptome in Wahrheit auf den Einsatz von Senfgas zurückzuführen seien ${ }^{46}$. Auf Plakaten, die Abdelkrim im Protektorat verteilen ließ, klagte er die Praktiken der Spanier an. Gleichzeitig ließ er die Rifbevölkerung vor den Folgen warnen ${ }^{47}$. Versuche ägyptischer Sympathisanten in Berlin, die Weltöffentlichkeit aufzurütteln, stießen jedoch auf geringe Beachtung ${ }^{48}$.

Spaniens Alliierter Frankreich verzichtete auf die Verwendung von Lost, hatte aber seit Beginn des Rifkrieges im Jahre 1921 Chemikalien an eine Gasfabrik bei Melilla geliefert ${ }^{49}$. Im Januar 1925 mokierte sich Marschall Lyautey noch über die spanischen Gasangriffe auf die Anjera ${ }^{50}$. Nach dem Angriff der Rifkabylen auf die französische Zone jedoch forderte er wegen der mehr als ernsten Lage nicht nur Verstärkungen, sondern auch Senfgasbomben an. Giftwaffen seien nötig, um Französisch-Marokko zu retten, schrieb Lyautey im Juni 1925 an Ministerpräsident Paul Painlevé. Dieser erteilte zwar keine

\footnotetext{
${ }^{41}$ MARTINETZ, Giftpfeil, S. 157.

${ }^{42}$ BALFOUR, Deadly Embrace, S. 129.

${ }^{43} \mathrm{KUNZ}$, 》Con ayuda«, S. 181.

44 SHAT 3H1559: Commissariat de la sûreté générale Oujda an Consul général de France Oujda, 20.12.1923.

${ }^{45}$ WOOLMAN, Rebels in the Rif, S. 186, S. 207; ABDELKRIM, Memoiren, S. 113; SHAT 3H442: Note de renseignements o.D. [Mitte 1926] nach Aussagen des deutschen Deserteurs Karl Ohme; Le typhus décimerait les Rifains? Abd el Krim demanderait aide sanitaire, in: Petit Journal, 9.4.1926.

${ }^{46}$ KUNZ, MǗlLER, Giftgas, S. 32, S. 126-128; MARTINETZ, Giftpfeil, S. 158.

${ }^{47} \mathrm{KUNZ}$, 》Con ayuda«, S. 178; BALFOUR, Deadly Embrace, S. 152.

${ }^{48}$ Siehe hierzu Kapitel 6, S. 313.

${ }^{49}$ BALFOUR, Deadly Embrace, S. 128, S. 133f.

${ }^{50}$ Daniel RIVET, Le commandement français et ses réactions vis-à-vis du mouvement rifain 1924-1926, in: Abd el-Krim et la République du Rif, S. 101-136, S. 103.
} 
Erlaubnis für deren Einsatz, rechtfertigte aber jedes sonstige Mittel ${ }^{51}$. Frankreich beschränkte sich - und selbst dies wurde dementiert ${ }^{52}$ - auf die Verwendung von Tränengas ${ }^{53}$. Die französische Regierung duldete die spanischen Praktiken ohne Kritik. Sie hatte vom spanisch-deutschen Gasgeschäft Kenntnis, ohne dagegen einzuschreiten, denn sie setzte auf eine möglichst schnelle Beendigung des Krieges ${ }^{54}$. Auch der britische Geheimdienst wußte vom spanischen Gaseinsatz. Großbritanniens Regierung wollte Spaniens Situation in Marokko gestärkt sehen. Hier ging es um übergeordnete britische Interessen im mediterranen Raum ${ }^{55}$. Das Völkerbundprotokoll vom 17. Juni 1925, das - während der Hochphase der spanischen Gasangriffe - den Ersteinsatz chemischer Waffen untersagte und sowohl von Frankreich als auch von Spanien ratifiziert wurde, war somit Makulatur ${ }^{56}$.

Gegen Giftgas war die Rifbevölkerung während des Krieges völlig schutzlos. Weder dessen Wirkung noch Gegenmaßnahmen waren bekannt. Es wurde vergeblich versucht, Senfgaswunden durch das Aufgießen von heißem Öl rauszubrennen<. Eine Entgiftung war mit den bescheidenen Mitteln der Rifkabylen nur durch Verbrennung von allem, was verseucht war, möglich ${ }^{57}$. Versuche der Riftruppen, ihre Unterstände mit feuchten Decken gegen Giftgase zu schützen, waren ebenso nutzlos wie die Anfertigung von Ledermasken durch spanische Kriegsgefangene ${ }^{58}$. Vielen blieb nur die Flucht vor »sim«, wie Senfgas im Rif genannt wurde ${ }^{59} .6000$ bis 7000 Flüchtlinge hatte die neutrale Stadt Tanger bis August 1925 aufzunehmen ${ }^{60}$. Bis Juli 1927, als die letzten Widerstandskämpfer im Rif aufgaben, setzten die spanischen Truppen Gas ein $^{61}$.

${ }^{51}$ HOISINGTON, Lyautey, S. 196, S. 198.

${ }^{52} \mathrm{~L}$ 'armée française n'a pas fait usage de gaz asphyxiants, in: Matin, 21.7.1925.

${ }^{53}$ MARTINETZ, Giftpfeil, S. 159; KUNZ, MüLlER, Giftgas, S. 20; KUNZ, "Con ayuda“, S. 164; Otto WELSCH, Das marokkanische Problem, in: Militär-Wochenblatt, 4.7.1925; Erich LILIENTHAL, Giftgas in Marokko, in: Tägliche Rundschau, 5.6.1925.

${ }^{54}$ ADMAE Maroc 198, 19: Ambassade de Madrid, Attaché militaire an MAE, 30.4.1924.

${ }^{55} \mathrm{Vgl}$. insbesondere den detaillierten Bericht des Madrider Militärattachés W.W.T. Torr in: PRO-FO 371, 11077, 39, 40: Embassy of Madrid an FO, 20.5.1925.

${ }^{56}$ STLLLR, Gaskrieg, S. 41 f.; BALfOUR, Deadly Embrace, S. 127f.; KUNZ, MÜLLER, Giftgas, S. 23, S. 69; BRIEDEN, Giftgasbomben, 17.7.1998; MARTINETZ, Giftpfeil, S. 159.

${ }^{77}$ KUNZ, MǗLLER, Giftgas, S. 58, S. 125, S. 158; KUNZ, "Con ayuda《, S. 177.

${ }^{58}$ SPENCER PRYSE, Through the Lines, S. 620; SHAT 3H1557: Territoire de Taza, Cercle de Guercif, SR, Bericht »Renseignements fournis sur le Riff«, 8.2.1925.

${ }^{59}$ BALFOUR, Deadly Embrace, S. 154.

${ }^{60}$ PRO-FO 371, 11921, 7472: CG Tangier an FO, 1.8.1926 mit dem Bericht "Tangier, Annual Report, 1925-1926«, S. 8; Anna R. FRY, Tangier To-day, in: Foreign Affairs 7 (1925-1926) S. 68f., S. 69; Charles HOBHOUSE, Relieving Refugees from the Riff, in: Manchester Guardian, 1.9.1925; Muhammad Y. ABBASI, London Muslim League 19081928. An Historical Study, Islamabad 1988, S. 379.

${ }^{61}$ KUNZ, MŨLLER, Giftgas, S. 171f.; BRIEDEN, Giftgasbomben, 17.7.1998. 
Der Gaskrieg trug maßgeblich zur Niederlage der Rifkabylen bei. Die Folgen sind bis heute spürbar. Im Sommer 2000 wurde in Marokko eine Studie des Rabater Centre hospitalier universitaire (CHU) bekannt. Sie brachte die Tatsache, daß 60\% der Kehlkopfkrebs-Toten Marokkos aus der Region Nador stammen, direkt mit den von Spanien mehr als 70 Jahre zuvor im Rif eingesetzten Chemiewaffen in Verbindung. "Akhenzir« nennt man im Rif diese Krankheit. Am 8. Juli 2000 gründete der marokkanische Menschenrechtler Ilyas el Omari in Alhucemas die Association de défense des victimes du gaz toxique dans le Rif. Sie setzt sich zum Ziel, mit Hilfe von Schadenersatzansprüchen gegen Spanien, Frankreich und Deutschland den heutigen Kranken zu helfen. Die marokkanische Regierung, die seit dem Rifaufstand von 1958 bis 1959 eine Politik der Marginalisierung der Berberbevölkerung verfolgt, verbot 2001 zunächst von Omari organisierte Tagungen, auf denen Wissenschaftler über den Gaskrieg referieren wollten. Vermutlich standen wegen des angespannten Verhältnisses zwischen Marokko und Spanien diplomatische Gründe dahinter. Keine spanische Regierung bekannte sich bisher zum Giftgaseinsatz im Rif ${ }^{62}$. Ähnlich erklärte das deutsche Auswärtige Amt auf Anfrage, »das menschliche Leid und die Tragödie der Opfer« zu bedauern, es gebe jedoch »keine rechtlichen Grundlagen für Schadenersatzansprüche $\ll^{63}$. Auf einer von der Zeitung "Le monde amazigh« vom 14. bis zum 15. Februar 2004 veranstalteten wissenschaftlichen Fachtagung in Nador, an der unter anderem auch Sebastian Balfour, Ángel Viñas, Juan Pando und Maria-Rosa de Madariaga teilnahmen, wurden die Spätfolgen der spanischen Gasangriffe bestätigt $^{64}$.

${ }^{62}$ BALFOUR, Deadly Embrace, S. 129, S. 155f.; KUNZ, "Con ayuda«, S. 184; Abdallah NAANA, Quand les gaz toxiques arrosaient le Rif, in: La vie économique, 28.7.-3.8.2000; Lothar A. HENRICH, Die Schlacht von Anoual vor achtzig Jahren, in: Junge Welt, 21.7.2001; Reiner WANDLER, Deutsch vergast, marokkanisch vergessen, in: Tageszeitung, 26.1.2002.

${ }^{63}$ Benachrichtigung von Jens Krauss-Massé, 19.11.2001.

${ }^{64}$ Hebert ABD-EL KRIM, Guerre chimique pendant la guerre du Rif sur le satellite ALAARABIA, in: Antiweblog, 13.3.2004. URL: http:/www.preavis.org/antiweblog/article.php 3?id_article=52 [16.1.2005]; Paco SOTO, El ejército colonial español empleó armas químicas en la guerra del Rif, in: Andalucia libre $\mathrm{N}^{\circ} 223,23.2 .2004$. URL: http://www.elistas.net/ lista/andalucialibre/archivo/indice/201/msg/258 [16.1.2005]; Colloque sur les armes chimiques pendant la guerre du RIF, in: Kabyle.com, 15.2.2004. URL: http:/www.kabyle.com/ article.php3?id_article=8297 [16.1.2005]; Information von Mimoun Charqi, 8.12.2004. 


\subsection{Abdelkrims Kontakte nach London und Algerien}

Festzuhalten bleibt, daß seit 1921 bei allen beteiligten Kampfverbänden humanitäre Grenzen überschritten und Hemmschwellen immer weiter gesenkt wurden. Der Gedanke an Rache war auf allen Seiten ausgeprägt. Insbesondere die Zivilbevölkerung hatte darunter zu leiden. Abdelkrim erkannte schnell, daß humanitäre Hilfsleistungen aus dem Ausland unbedingt erforderlich waren, denn im Rif gab es weder Ärzte noch Medikamente. Es fehlte an allem. Verwundete Rifsoldaten mußten nach traditionellen Methoden behandelt werden. Heiße Asche oder Spinnweben sollten Blutungen stillen. Kugeln wurden ohne Betäubung herausgeschnitten ${ }^{65}$. »Die einzigen Ärzte sind eingeborene Pflanzensammler und Heilgehilfen mit unbrauchbaren Operations-Geräten ${ }^{66}$, beobachtete der Rifbesucher Gerald Spencer Pryse Ende 1924. Es gab kein Pflegepersonal für Verwundete, die in der Regel an Wundbrand starben. Eine Art Gesundheitsdienst existierte zunächst nicht ${ }^{67}$. Die 1921 in Annual erbeuteten Medikamente waren schnell aufgebraucht. Ärzte und Arzneien wurden dringend benötigt ${ }^{68}$. Der einzige Ausweg war, von auswärts Hilfe zu holen.

Der erste europäische Mediziner im Rif war allerdings unfreiwillig dort. Während der Kämpfe bei Annual und Jebel Aroui im Sommer 1921 war der spanische Arzt Antonio Vazquez Bernaescu in Gefangenschaft geraten. Als persönlicher Gefangener Abdelkrims in Ajdir hatte Vazquez die umliegenden Dörfer medizinisch zu versorgen. Das Angebot, für eine hohe Summe Geld als Arzt für die Riftruppen zu arbeiten, schlug er aus. Vazquez zog die Flucht vor, indem er Ende September 1921 vom Strand der Bucht von Alhucemas aus zu einem spanischen Schiff schwamm ${ }^{69}$. Sein Kollege Serrano beschränkte seine Aktivitäten auf das spanische Gefangenenlager ${ }^{70}$. Die beiden Spanier waren für lange Zeit die letzten europäischen Ärzte, die sich im Rif aufhielten.

An Bemühungen Abdelkrims, Hilfe zu holen, mangelte es jedoch nicht. Einer seiner Vermittler war der Brite John Arnall. Nach einer glücklosen Karriere als Politiker der Independent Labour Party und als Besitzer eines Fotogeschäftes in Marseille waren John und seine Ehefrau Gertrude Arnall 1919 nach

\footnotetext{
${ }^{65}$ WOOLMan, Rebels in the Rif, S. 185; PenNell, A Country, S. 150; KUNZ, Mưller, Giftgas, S. 122; HARRIS, France, S. 299.

${ }^{66}$ SPENCER PRYSE, Through the Lines, S. 644.

${ }^{67}$ Walter B. HARRIS, Conditions in the Rif. English Travellers' Report, in: Times, 3.12.1924; PRO-FO 371, 8354, 4882: John Arnall an Arthur Field, 29.5.1922; CADN Maroc DAI 187: RG Rabat an MAE, 22.12.1923; Guy de MONTJOU, La Guerre du Rif, in: Revue de Paris 32 (1925) S. 574-601, S. 593f.

${ }^{68}$ ABDELKRIM, Memoiren, S. 95; Youssoufl, Les institutions, S. 98.

${ }^{69}$ Captive of Moors Escapes, in: New York Times, 2.10.1921.

${ }^{70}$ GANTE, El cautiverio, 8.1.1922.
} 
Tanger übergesiedelt. Seit 1913 bereits verbanden sie politische und geschäftliche Interessen (Bergbau, Schmuggel) mit dem Rif ${ }^{71}$. Arnalls humanitäres Engagement ist vor dem Hintergrund dieser Geschäftsbeziehungen zu relativieren. Während eines Aufenthaltes in London Ende August 1921 appellierte er an das britische Außenministerium, verwundeten Rifsoldaten und der Zivilbevölkerung im Rif die dringend benötigte medizinische Hilfe zukommen zu lassen $^{72}$. Doch die britische Regierung verhielt sich während des gesamten Krieges strikt neutral und wollte sich auf keinen Fall in die Angelegenheiten Spanisch-Marokkos einmischen ${ }^{73}$. Nicht einmal eine Ende 1921 von Spanien erbetene Hilfsmission des britischen Roten Kreuzes für die spanischen Gefangenen der Rifkabylen kam für das Außenministerium in Frage - wegen der Befürchtung, die britischen Helfer könnten von den Rifkabylen als offizielle Repräsentanten Großbritanniens aufgefaßt werden ${ }^{74}$.

Das Einzige, was Arnall in humanitärer Hinsicht in London erreichte, war, über seinen Freund Arthur Field von der Anglo-Turkish Society Kontakt zur British Red Crescent Society aufzunehmen. Die humanitäre Organisation war 1911 vom indischen Juristen Syed Ameer Ali - ebenso Gründer der London Muslim League (London Branch of All India Muslim League) - ins Leben gerufen worden. Während des Ersten Weltkrieges hatte die British Red Crescent Society indische Offiziere versorgt, die im Norden Frankreichs gekämpft hatten $^{75}$.

Zahlreiche britische Moslems oder Moslemfreunde engagierten sich in ihr, wie etwa Charles Wallace Alexander Napier Ross Cochrane-Baillie alias Lord Lamington. Der Brite, der 1890 bis 1891 eine vielbeachtete Reise von Siam nach Tongking unternommen hatte (»Journey through the Trans-Salween Shan States to Tong King«, 1891), war von 1896 bis 1901 Gouverneur im australi-

${ }^{71}$ Siehe zu Arnalls politischen und wirtschaftichen Interessen Kapitel 5, S. 172f., S. 198 und Kapitel 6, S. 264-285.

${ }^{2}$ PRO-FO 371, 7082, 8256: John Arnall an FO, 30.8.1921 und 6.9.1921.

${ }^{73}$ PRO-FO 371, 7082, 8256: Note, 2.9.1921.

${ }^{74}$ Spanish Prisoners' Ransom, in: Times, 9.12.1921; PRO-FO 371, 7086, 12777: Embassy of Madrid an FO, 16.12.1921. Siehe zur Haltung der britischen Regierung insbesondere Kapitel 6, S. 264-305 und S. 316-354.

${ }_{75}$ ABBASI, London Muslim League, S. 13-21, S. 281, S. 385; DERS., Leadership of London Muslim League. Analysis of the Tussle between Mohamed Ali Johar and Syed Ameer Ali, in: Journal of the Pakistan Historical Society 32 (1984) S. 1-12, S. 2, S. 8; Ali A. KHAN, Ameer Ali's Services to Turkey, in: Journal of the Asiatic Society of Bangladesh 22 (1977) S. 54-65, S. 55; Khursheed K. AzIZ, Ameer Ali. His Life and Work, Lahore 1968, S. 2-12, S. 15, S. 20; Shan MuhammaD, The Right Honourable Syed Ameer Ali. Personality and Achievements, Neu-Dehli 1991, S. 143, S. 188f.; Muhammad N. QURESH, Mohamed Ali's Khilafat Delegation to Europe (February-October 1920), Karachi 1980, S. 17; S.V. FITZGERALD, Syed Ameer Ali 1849-1928, in: John R.H. WEAVER (Hg.), The Dictionary of National Biography 1922-1930, Oxford 1937, S. 18f.; Muhammad A. RAHIM, Syed Ameer Ali and the Muslim Renaissance Movement, Chittagong ${ }^{2} 1980$, S. 25. 
schen Queensland und von 1903 bis 1907 Gouverneur von Bombay, ehe er dann nach einem Syrien-Aufenthalt (1919) im House of Lords saß. Er gehörte vielen Organisationen an, die sich mit orientalischen Fragen befaßten, und galt als Verfechter der Rechte von Minderheiten und kleineren Nationen ${ }^{76}$. Weitere namhafte Mitglieder waren Cuthbert Francis Dixon-Johnson und Ernest Harrison Griffin. Sie hatten an einer Delegation der British Red Crescent Society teilgenommen, die im Zuge der italienischen Annexion von Tripolitanien (1911-1912) die türkische Armee humanitär versorgt hatte ${ }^{77}$. Griffin war als eifriger Sympathisant moslemischer Völker bekannt ${ }^{78}$. Zu nennen wäre ebenfalls Charles Foster Ryder. Er hatte 1913 zusammen mit John Arnalls Freund Arthur Field die Anglo-Ottoman Society und 1917 die Workers Welfare League for India gegründet ${ }^{79}$. Field, Dixon-Johnson und Ryder wurden später (1925) Mitglieder des Londoner Riff-Committees, um politische Unterstützungsarbeit für Abdelkrim zu leisten ${ }^{80}$.

Londons moslemische Gemeinschaft war durch hoch politisierte Studenten aus Indien zahlenmäßig angewachsen ${ }^{81}$. Die Initiative, den Glaubensbrüdern im Rif humanitär zu helfen, war vom Londoner Zweig der pan-islamistischen All India Muslim League ausgegangen, dessen ehrenamtlicher Geschäftsführer M. Hashim Isaphani zusammen mit Mushir Hosain Kidwai von der Central Islamic Society und mit Marmaduke Pickthall auch das Islamic Information Bureau - das Zentrum pan-islamistischer Propaganda in Großbritannien - leitete $^{82}$. Die Ideologie des Pan-Islamismus verfolgte zu dieser Zeit das Ziel, die moslemische Welt unter dem gemeinsamen Band des islamischen Glaubens zu vereinen ${ }^{83}$.

${ }^{76}$ A. COCHRANe, Charles Wallace Alexander Napier Ross Cochrane-Baillie, Second Baron Lamington 1860-1940, in: Loopold G.W. LEGG (Hg.), The Dictionary of National Biography, Bd. 4: 1931-1940, London 1949, S. 30f.

7 ABBASI, London Muslim League, S. 283f., S. 390; MUHAMmaD, Syed Ameer Ali, S. 143; ADMAE Maroc 133, 233: Consul de Glasgow an MAE, 23.2.1926; Ernest H. GRIFFIN, Adventures in Tripoli. A Doctor in the Desert, London 1924.

${ }^{78}$ IORL, P\&J, 127230, 1671 (1924): A. Merry del Val an Lord Birkenhead, Secretary of State for India, 18.3.1925.

${ }^{79}$ PRO-FO 371, 2344, 2889: Eingabe $» P e t i t i o n$ for a Royal Charter, Request for the Grant of a Charter of Incorporation to the British Red Crescent Society«, 9.12.1926; Sehri SAKLATVALA, The Fifth Commandment. A Biography of Shapurii Saklatvala, Kalkutta 1996, S. 77; Anne FrEEMANTLE, Loyal Enemy, London 1938, S. 228.

${ }^{80}$ Siehe zum Riff-Committee Kapitel 6, S. 316-346.

${ }^{81}$ Peter CLARK, Marmaduke Pickthall. British Muslim, London u.a. 1986, S. 39.

${ }^{82}$ QURESHI, Khilafat Delegation, S. 17f. Vgl. S. Tanvir WASTI, Mushir Hosain Kidwai and the Ottoman Cause, in: Middle Eastern Studies 30 (1994) S. 252-261.

${ }^{83}$ Edmund BURKE, Pan-Islam and Moroccan Resistance to French Colonial Penetration 1900-1912, in: Journal of African History 13 (1972) S. 97-118, S. 98; Jacob M. LANDAU, The Politics of Pan-Islam. Ideology and Organization, Oxford 1994, S. 5f. 
Anfang 1922 schickte Isaphani einen Brief an einen reichen Geschäftsmann in Bombay namens Seth Mian Muhammad Haj Jan Muhammad Chotani ${ }^{84}$, den Präsidenten des All India Central Khilafat Committee. Er rief ihn auf, sich um Ärzte, Medikamente und Kleidung für die Rifkabylen in Marokko zu kümmern $^{85}$. Am 14. März 1922 wandte sich Chotani an Syed Ameer Ali in London, um über ihn beim britischen Außenministerium die Erlaubnis zu erbitten, von Indien aus eine ausschließlich aus moslemischen Ärzten bestehende Mission unter der Führung der British Red Crescent Society ins Rif schicken zu dürfen. Lord Lamingtons Anfrage vom 19. März 1922 wurde negativ beschie$\operatorname{den}^{86}$.

John Arnall teilte Abdelkrim die vagen Pläne einer humanitären Mission während eines Aufenthaltes im Rif von April bis Mai 1922 mit. Dieser untermauerte den dringenden Bedarf an Ärzten und Medikamenten ${ }^{87}$. Sie kamen überein, daß Arnall eine Rif-Delegation nach London begleiten sollte, die von dort aus in der moslemischen Welt um humanitäre Unterstützung bitten und direkt mit der British Red Crescent Society in Kontakt treten sollte ${ }^{88}$. Esme Howard, der britische Botschafter in Madrid, warnte davor, daß eine Hilfsaktion des britischen Roten Kreuzes oder Halbmondes ins Rif einen schlechten Eindruck in Spanien hinterlassen würde, zumal eine derartige Mission zu den spanischen Gefangenen bereits von der britischen Regierung abgelehnt worden sei. Im Außenministerium stimmte man dieser Ansicht $\mathrm{zu}^{89}$.

Von dort hatten die Rifemissäre Si Mohammed Boujibar und Si Abdelkrim ben el Haj Ali Luh (Ali Luh), die Großbritannien am 10. Juli 1922 erreichten, daher keine Hilfe zu erwarten. Das völlige Abblocken der britischen Regierung, selbst was medizinische Hilfe für das Rif anbetraf, setzte den Möglichkeiten der British Red Crescent Society enge Grenzen. Nach einer Unterredung mit den Rifdelegierten traf sich Syed Ameer Ali am 19. Juli 1922 mit Spaniens Botschafter in London Alfonso Merry del Val. Er versuchte, das Schicksal der spanischen Kriegsgefangenen im Rif mit der humanitären Hilfe für die Rifbevölkerung zu verbinden. Er hatte jedoch genauso wenig Erfolg

${ }^{84}$ QURESHI, Khilafat Delegation, S. 1f.

${ }^{85}$ India Asked to Help Morocco, in: The Moslem World 12 (1922) S. $305 f$.

${ }^{86}$ PRO-FO 371, 8353, 2435: Lord Lamington an FO, 19.3.1922; FO an Lord Lamington, 24.3.1922 und 3.4.1922.

${ }^{87}$ CADN Tanger A427: Centre de renseignements extérieurs du Maroc, Tanger an CG Tanger, 12.6.1922; PRO-FO 371, 8354, 4882: John Arnall an Arthur Field, 29.5.1922; PRO-FO 371, 8354, 4882: New Scotland Yard, Special Branch an FO, 10.6.1922.

${ }^{88}$ PRO-FO 371, 8354, 4882: John Arnall an Arthur Field, 30.5.1922; PRO-FO 371, 8342, 171: Bericht »Memorandum by Mr Wingfield on the Moroccan Situation«, 5.7.1922; MADARIAGA, L'Espagne et le Rif, Bd. 2, S. 205; Walter B. HARRIS, A Rifi Appeal, in: Times, 26.6.1922. Siehe zu den politischen und wirtschaftlichen Aspekten der Mission Kapitel 6, S. 264-281.

${ }^{89}$ PRO-FO 371, 8354, 4882: Embassy of Madrid an FO, 7.7.1922; Note, 11.7.1922. 
wie Ernest H. Griffin mit seiner Eingabe im darauffolgenden August ${ }^{90}$. Die Initiativen der British Red Crescent Society verliefen somit zunächst im Sande und wurden erst 1924 wieder aufgenommen. Die Gesandten aus dem Rif reisten im Dezember 1922 ab, ohne viel erreicht zu haben.

Spärliche Hilfsangebote für die Rifkabylen kamen aus Algerien. Der Arzt Abdelaziz ben Mourad Tourki aus Algier hielt sich im Sommer 1922 im Rif auf $^{91}$. Im Oktober 1923 litt eine Schwester Abdelkrims, die mit Außenminister Si Mohammed Azerkane verheiratet war, schwer an den Folgen einer komplizierten Geburt ${ }^{92}$. Abdelkrim ließ nichts unversucht, sofort Fachkräfte zu holen. Als Caïd Haddou mit dem algerischen Arzt Zorbaïdes Taourirt erreichte, wurde ihnen vom französischen Militär die Einreise ins Rif verweigert ${ }^{93}$. Abdelkrim, der eigentlich gute Beziehungen zum französischen Nachbarn zu pflegen glaubte, war wegen dieser Maßnahme sehr verbittert. Anfang November 1923 wurde Caïd Haddou von Abdelkrim erneut nach Oujda geschickt, um den früheren Militärarzt und nunmehrigen Geschäftsmann Dr. Yves Marion-Gallois und die Hebamme Mlle Ponzo mit dem Auto nach Taourirt und dann zu Pferd nach Ajdir zu bringen ${ }^{94}$. Es mögen auch humanitäre Gründe gewesen sein, die die Generalresidenz in Rabat diesmal dazu bewogen, die Hilfsmission zu gestatten ${ }^{95}$. Sicher spielte der Gedanke, die Beziehungen zu Abdelkrim nicht zu verschlechtern oder zu gefährden, die wichtigere Rolle. Marschall Lyautey verfolgte zu dieser Zeit eine von Ambiguität geprägte Politik gegenüber dem Rif $^{\text {f6. }}$.

Am 7. November 1923 kamen Marion-Gallois und Mlle Ponzo in Ajdir an. Zur Überraschung des Arztes ritt die Hebamme allein zur Kranken nach Aït Kamara weiter. Als Mann durfte Marion-Gallois die Schwester Abdelkrims, die zudem der europäischen Medizin sehr mißtraute, nicht selbst untersuchen.

${ }^{90}$ IORL, P\&J, 12, 230, 1671 (1924): A. Merry del Val an Lord Birkenhead, Secretary of State for India, 18.3.1925.

91 ADMAE Maroc 194, 216: CG Tanger an MAE, 8.7.1922; CADN Tanger A427: Note o.D. (Juni 1922).

${ }_{92}$ DAOUD, Abdelkrim, S. 174; Pierre FONTAINE, L'étrange aventure riffaine, Paris 1943, S. 59, S. 61f.; FURNEAUX, Abdel Krim, S. 92. Alle geben den falschen Monat an (März 1923).

${ }^{93}$ SHAT 3H1559: Commissariat de la sûreté régionale Oujda, Renseignements, 19.10.1923. Der Bericht gibt keinen Grund dafür an, warum die Einreise verweigert wurde. Vermutlich hatte dies damit zu tun, daß man Gerüchten entgegentreten wollte, Frankreich unterstütze heimlich die Rifkabylen [CADN Madrid C274: Ambassade de Madrid an MAE, 24.8.1923 (Bericht über erboste spanische Presseberichte); The Rif Rising. French Deny Spanish Charges, in: Times, 11.8.1923; PRO-FO 371, 9470, 44, 126: CG Tangier an FO, 13.10.1923].

${ }^{94}$ CADN Maroc DAI 187: RG Rabat an MAE, 22.12.1923; FONTAINE, L'étrange aventure, S. 59; DERS., Abd el-Krim, S. 97; Roger-MATHIEU, Mémoires, S. 226.

${ }_{95}$ CADN Maroc DAI 187: RG Rabat an MAE, 22.12.1923.

${ }^{*}$ GERSHOVICH, French Military Rule, S. 126f. Siehe zur französischen Haltung insbesondere Kapitel 5, S. 160-169, S. 192-197 und Kapitel 6, S. 241-264, S. 334-343. 
Per Telefonverbindung war er mit dem zehn Kilometer entfernten Aït Kamara verbunden, um die Diagnose zu stellen, zu behandeln, den Verlauf der Erkrankung zu verfolgen und auf Komplikationen zu reagieren. Er schaffte es schnell, die Kranke außer Gefahr zu bringen. Marion-Gallois und Mlle Ponzo blieben zur Vorsicht noch bis zum Dezember 1923 im Rif. Beide erhielten hohe Belohnungen. Mlle Ponzo kehrte im Mai 1924 noch einmal ins Rif zurück, um während einer schweren Pockenepidemie mit Impfungen zu helfen. Die zur Verfügung stehenden Impfflüssigkeiten reichten aber nur für drei Wochen $^{97}$.

\subsection{Das Internationale Komitee des Roten Kreuzes und die Aktivitäten der British Red Crescent Society}

Das Internationale Komitee des Roten Kreuzes (IKRK) in Genf - seit 1863 das organisatorische Bindeglied der nationalen Rotkreuz-Gesellschaften hatte sich bisher nicht zum Rifkrieg geäußert. Dies entsprach der damals selbst auferlegten Einschränkung, nur auf ausdrückliche Anfrage hin Maßnahmen einzuleiten. Über die Frage, ob das Rote Kreuz im Falle einer Rebellion in einer Kolonie oder in einem Protektorat wie Marokko überhaupt zuständig war, herrschten zwischen dem IKRK und den Kolonialstaaten unterschiedliche Auffassungen. Dies demonstrierte der Rifkrieg beispielhaft ${ }^{98}$. Die erste internationale Konferenz des Roten Kreuzes nach dem Ersten Weltkrieg im Jahre 1921 hatte in einer Resolution betont, daß auch die Opfer von Bürgerkriegen nicht nur die Opfer zwischenstaatlicher Konflikte - ein Anrecht auf Hilfe hätten. Wenn nationale Rotkreuz-Gesellschaften nicht allen Bürgerkriegsparteien in gleicher Weise helfen konnten, sollte das IKRK als neutrale Zwischeninstanz dienen und mit der Zustimmung der Regierung des sich im Bürgerkrieg befindlichen Landes andere neutrale Gesellschaften um Unterstützung

${ }^{97}$ FonTANE, L'étrange aventure, S. 58f., S. 61f., S. 65f.; DAOUD, Abdelkrim, S. 193; SHEEAN, My Meeting, S. 852; SHAT 3H1559: Annexe de contrôle civil d'El Aïoun, Bulletin de renseignements, 17.11.1923; CADN Maroc DAI 187: RG Rabat an MAE, 22.12.1923 mit dem Bericht von Dr. Marion-Gallois »Relations de voyage dans le Riff«, 8.12.1923; SHAT 3H1102: Territoire de Taza, Cercle de Guercif, SR, Feuille de renseignements, 12.12.1923; CADN Tanger C38: Région de Fès, SR, Bulletin de renseignements, 17.12.1925.

${ }^{98}$ André DuRAND, Histoire du Comité international de la Croix-Rouge, Bd. 2: De Sarajevo à Hiroshima, Genf 1978, S. 198. 
bitten dürfen. Innerhalb des IKRK sah man zwischen einer kolonialen Rebellion und einem Bürgerkrieg keinen Unterschied. Niemand in Genf betrachtete den Rifkrieg als zwischenstaatlichen Konflikt ${ }^{99}$.

Am 12. Juli 1924 erhielt das IKRK in Genf ein Schreiben von der Londoner Near and Middle East Association. Die streng pro-türkische Vereinigung war im November 1921 gegründet worden. Ihr gehörten namhafte Parlamentsabgeordnete wie Aubrey Herbert oder Josiah Wedgwood an. Syed Ameer Ali, Lord Lamington, Cuthbert F. Dixon-Johnson und Ernest H. Griffin von der British Red Crescent Society waren Mitglieder. Edward Gleichen stand der Gesellschaft vor ${ }^{100}$. Als ehrenamtlicher Generalsekretär fungierte Captain Ernest Nathaniel Bennett ${ }^{101}$. Bennetts Brief forderte das IKRK angesichts des Leidens der Menschen im Rif auf, eine Hilfsmission zu entsenden. Da das spanische Rote Kreuz für Spanisch-Marokko zuständig war, leitete das IKRK den Brief nach Madrid weiter. Ob dies im Interesse Abdelkrims war, muß bezweifelt werden. Seine Hilfsappelle richteten sich nie an seinen Kriegsgegner Spanien. Am 1. August 1924 kam die Antwort des spanischen Roten Kreuzes, daß im Einklang mit der spanischen und mit der marokkanischen Regierung die Meinung vorherrsche, eine Hilfsmission zu den marokkanischen »Rebellen« sei angesichts der laufenden "polizeilichen Operationen zur Aufrechterhaltung der Ordnung « nicht opportun ${ }^{102}$.

Die Betonung von Polizeioperationen in Marokko unterstrich das Bemühen, die Ereignisse als nichtkriegerische Akte gegen nicht kriegführende Rebellen darzustellen. Auf diese Weise betrachtete sich das spanische Rote Kreuz als nicht zuständig. Seit 1921 betrieb es indes Hospitäler in Melilla, Ceuta, Tetuan, Larache und Melilla. Mit dem Gesundheitsdienst des spanischen Militärs arbeitete es an verschiedenen Frontabschnitten - z.B. Dar Driouch, Tafersit und Nador - zusammen ${ }^{103}$. Rotkreuz-Hospitäler in San José and Santa Adéla behandelten verwundete spanische Soldaten. In Spanien führten Frauen des Roten Kreuzes Sammlungen für die Soldaten in Afrika durch ${ }^{104}$. Spanischen

${ }^{99}$ Dieter RIESENBERGER, Für Humanität im Krieg und Frieden. Das Internationale Rote Kreuz 1863-1977, Göttingen 1992, S. 129; François BUGNION, Le Comité international de la Croix-Rouge et la protection des victimes de la guerre, Genf 1994, S. 338, S. 1180.

${ }^{100}$ Medical Supplies for the Rifi, in: Manchester Guardian, 25.11.1924.

${ }^{101}$ PRO-FO 371, 9114, 4046, 101: Near and Middle East Association an FO, 20.4.1923; PRO-FO 371, 9176, 10839, 64: Near and Middle East Association an FO, 1.11.1923; PROFO 371, 6581, 13103: Scotland House an FO, 26.11.1921. Zu Bennetts weiteren humanitären Aktivitäten gehörte die Mitgliedschaft zum German Distress Relief Fund [The Distress in Germany, in: Manchester Guardian, 27.10.1923].

${ }^{102}$ DURAND, Histoire, Bd. 2, S. 198; Demandes d'intervention. Secours aux blessés du Riff, in: BISCR 56 (1925) S. 113-116, S. 113.

${ }^{103}$ L'cuvre de la Croix-Rouge espagnole au Maroc, in: BISCR 57 (1926) S. 661-669, S. 661-663.

${ }^{104}$ Le Croix-Rouge espagnole et la campagne d'Afrique, in: BISCR 53 (1922) S. 257; Para los soldados de Africa, in: Blanco y Negro, 4.9.1921. 
Gefangenen im Rif wurde mit Unterstützung des spanischen Roten Kreuzes Kleidung zugesandt ${ }^{105}$. Die Weigerung, den Rifkabylen ebenfalls zu helfen, stand in krassem Gegensatz zur Haltung des IKRK und zu fundamentalen Prinzipien des Roten Kreuzes wie Neutralität und Unparteilichkeit. Doch solange die spanische Regierung keine Zustimmung für die Entsendung einer Hilfsmission ins Rif gab, konnte das IKRK nicht zur Tat schreiten. So blieb auch eine Bitte der British Red Crescent Society an die spanische Botschaft Londons, einen Hilfsappell an das spanische Rote Kreuz weiterzusenden, im September 1924 ohne Antwort ${ }^{106}$.

Syed Ameer Ali drängte das IKRK, angesichts der vor allem durch die Luftangriffe verursachten Leiden der Zivilbevölkerung nicht nachzugeben ${ }^{107}$. Also reiste im November 1924 der Generaldelegierte des IKRK Raymond Schlemmer - einer der Mitbegründer der französischen Pfadfinder-Bewegung - nach Madrid, um mit der spanischen Regierung und den spanischen Rotkreuzlern Gespräche zu führen. Ihm wurde dort klargemacht, daß Spanien als Protektoratsmacht des Sultans keinerlei internationale Einmischung - auch keine humanitäre Hilfe - dulden würde. In Paris war die französische Regierung nur mit der Zustimmung Spaniens zu einer Unterstützung bereit. Dies war zu diesem Zeitpunkt auszuschließen. Von daher konnte Schlemmer dem IKRK nur vermelden, daß die Entsendung einer Hilfsmission undurchführbar sei. Auch mehrere nationale Gesellschaften des Roten Kreuzes wandten sich nun mit der Aufforderung an ihre Dachorganisation, im Rif zu helfen. Das zuständige spanische Rote Kreuz lehnte es aber weiterhin ab, tätig zu werden. In einem Schreiben vom 4. März 1925 argumentierten die Spanier nun, daß die Bedingungen für die Ausübung des Dienstes in Marokko schlicht nicht vorhanden seien, da der marokkanische Sultan sich bisher geweigert habe, die Genfer Konventionen anzuerkennen. Wegen der im Rif vorherrschenden Anarchie und der chaotischen Verhältnisse seien Hilfsmaßnahmen unmöglich. Es handelte sich um eine Farce, da für die eigenen Truppen Hilfe geleistet wurde. Durch diese Haltung wurden auch die spanischen Gefangenen im Rif ihrem Schicksal überlassen. Es dauerte bis zum Oktober 1925, ehe das zur Untätigkeit verurteilte IKRK eine erneute Initiative startete ${ }^{108}$.

Die British Red Crescent Society wurde seit dem Sommer 1924 auch selbst wieder aktiver. Die treibende Kraft war der Brite Captain Robert Gordon-Canning. Eine Erbschaft hatte ihm finanzielle Unabhängigkeit beschert. Während

${ }^{105}$ ÁlVAREZ, The Betrothed of Death 2001, S. 209, Anm. 18; SABLOTNY, Légionnaire, S. 270.

${ }^{106}$ IORL, P\&J, 12, 230, 1671 (1924): A. Merry del Val an Lord Birkenhead, 18.3.1925.

${ }^{107}$ IORL, P\&S, 11, 259, 2500 (1925): Ameer Ali an Comité international de la Croix-Rouge, 16.9.1924.

${ }^{108}$ Demandes d'intervention. Secours aux blessés du Riff, in: BISCR 56 (1925) S. 114-116; La Croix Rouge et le Rif, in: BISCR 56 (1925) S. 941-948, S. 941 f. u. S. 941 Anm. 4; DuRAND, Histoire, Bd. 2, S. 198. 
seiner Militärzeit war er zwischen 1908 und 1919 mit den 10th Royal Hussars unter anderem nach Palästina und nach Indien gelangt. Hier wurde sein Interesse für die moslemische Welt geweckt ${ }^{109}$. Gordon-Canning nahm in der Folgezeit am Leben der Moschee im Londoner Stadtteil Woking teil, ohne aber der islamischen Religion beigetreten zu sein oder die arabische Sprache gelernt zu haben ${ }^{110}$. Er bewunderte die Stärke des Islam, unterschiedliche Kulturkreise und Nationen zu vereinen ${ }^{111}$ und betrachtete sich in seinem Selbstverständnis als "Sympathisant, Bewunderer und Bewahrer des Islam ${ }^{112}$. Gordon-Canning wollte als eine Art neuer »Lawrence von Arabien« eine Rolle in der internationalen Politik spielen ${ }^{113}$. Der Exzentriker galt kritischen Zeitgenossen als indiskreter und narzistischer Abenteurer, der um jeden Preis - auch feindlich gesinnte - Aufmerksamkeit zu erheischen suchte ${ }^{114}$.

Während eines achtmonatigen Tangeraufenthaltes in den Jahren 1923 und 1924 hatte Gordon-Canning John Arnall und seine Ehefrau Gertrude kennengelernt, die in ihm Sympathien für den Kampf der Rifkabylen weckten ${ }^{115}$. Über sie kam er in London mit der British Red Crescent Society in Kontakt. Anfang September 1924 - parallel zu deren Anfrage beim Genfer IKRK vom 16. September 1924 - sprach Gordon-Canning im Auftrag von Syed Ameer Ali im Außenministerium vor. Er bat für eine geplante Hilfsmission ins Rif um diplomatische Unterstützung bei den Protektoratsmächten Frankreich und Spanien. Dies wurde erneut kategorisch abgelehnt ${ }^{116}$. Syed Ameer Ali bekräftigte in einem eigenen Brief vom 10. September 1924 die rein humanitären

${ }^{109}$ Colin Cross, The Fascists in Britain, London 1961, S. 172; Robert BENEWICK, Political Violence and Public Order. A Study of British Fascism, London 1969, S. 120; Richard GRIFFITHS, Patriotism Perverted. Captain Ramsay, the Right Club and British Anti-Semitism 1939-1940, London 1998, S. 26; Walter B. HARRIS, Abd-el-Krim's Offer to Treat. A British Intermediary, in: Times, 18.12.1925; Jacques ROGER-MATHIEU, Le capitaine Gordon-Canning porteur d'une lettre d'Abd el Krim est arrivé à Marseille, in: Matin, 22.12.1925. Siehe zu Gordon-Canning Kapitel 6, S. 316-346.

${ }^{110}$ Auguste TERRIER, Les »Frères de la côte» du Rif, in: BCAF 36 (1926) S. 38-45, S. 39; PRO-FO 371, 10584, 783, 180: CG Tangier an FO, 9.12.1924.

${ }^{111}$ Robert Gordon-CANNING, Peace Be Upon Us All, in: Islamic Review 12 (1924) S. 213216, S. $213 \mathrm{f}$.

${ }_{112}$ DERS., Correspondence (Leserbrief), in: Islamic Review 12 (1924) S. 219f., S. 219.

${ }^{113}$ PRO-FO 371, 11079, 39, 81: CG Tangier an Embassy of Madrid, 21.7.1925; BENEWICK, Political Violence, S. 120; Cross, The Fascists, S. 172; GrIFFTHS, Patriotism, S. 26.

${ }^{114}$ PRO-FO 371, 11083, 7118, 99: Minute, 25.7.1925; PRO-FO 371, 11083, 7118, 106: Minute, 13.8.1925; PRO-FO 371, 11907, 5, 49: Minute, 9.1.1926; PRO-FO 371, 12354, 8, 160: Minute o.D. [Februar 1927]; PRO-FO 371, 12380, 424, 128: Minute, 12.8.1927; TNLA, BNS, 1, Williams: Walter Harris an Harold Williams, 14.1.1926. Vgl. FleMNG, Primo de Rivera and Abd-el-Krim, S. 220; PENnell, A Country, S. 210; SÁNChEZ PéreZ, Abdelkrim, S. 153.

${ }^{115}$ ADMAE Maroc 135, 190: CG Tanger an MAE, 2.2.1926; PA-AA R71126, K430427: Botschaft London an AA, 5.5.1924.

${ }^{116}$ PRO-FO 371, 10586, 7382, 14: Minute, 18.10.1924. 
Absichten der Mission, die auch den spanischen Gefangenen Hilfe bringen würde ${ }^{117}$. Staatssekretär William Tyrell war zu keiner Unterstützung bereit, aber er deutete an: »Es steht natürlich allen Privatpersonen und privaten Gesellschaften frei, in der Presse um Spenden zu bitten ${ }^{118}$.

Die Führung der British Red Crescent Society nahm dies zum Anlaß, auf eigene Faust eine Mission unter der Leitung von Robert Gordon-Canning zu entsenden. Da die Gesellschaft über recht magere Eigenmittel verfügte, erschien in der "Times« vom 18. Oktober 1924 ein von Ameer Ali, Lord Lamington und Sultan Muhammad Shah Aga Khan III. - dem spirituellen Oberhaupt der schiitischen Ismaeliten-Diaspora - unterzeichneter Leserbrief. Unter dem Motto »Medical Mission to Riff-Land« baten sie um Spenden für eine humanitäre Hilfsmission, die auch den spanischen Gefangenen zugute kommen sollte ${ }^{119}$. Bis Januar 1925 stieg das Spendenkonto auf 528 Pfund und 56 Schilling an. Mindestens 442 Pfund davon waren von indischen Sympathisanten an den Riff Medical Relief Fund geschickt worden. Der Aga Khan hatte 50 Pfund gespendet, Ameer Ali und Cuthbert F. Dixon-Johnson von der British Red Crescent Society jeweils fünf Pfund ${ }^{120}$. Im April 1925 gingen von Prinz Omar Toussoun aus Ägypten 500 Pfund ein $^{121}$. Ein indischer Moslem namens Anik, der als Schatzmeister im Londoner Waldorf Hotel residierte, nahm die Gelder entgegen ${ }^{122}$.

Die British Red Crescent Society hatte mittlerweile innerhalb der britischen Gemeinschaft Tangers einen kleinen Kreis von Mitstreitern etabliert. Ihr offizieller Vertreter dort war der Schotte Captain S. Cyril Beresford Mundey, der direkt nach seiner Ankunft in Tanger im Jahre 1924 die Zeitung »Akhbar el Alam« gegründet hatte ${ }^{123}$. Erstmals machte Mundey in der Riffrage auf sich aufmerksam, als er in der Tangerer Zeitung "Al-Moghreb Al Aksa \& Tangier Gazette« einen Leserbrief veröffentlichte. Darin verdeutlichte er, daß Abdelkrim gerade deshalb keinen europäischen Standard in Bezug auf die Gesundheitsversorgung seiner Gefangenen halten könne, weil ihm Medizin, Zelthospitäler und Gebäude fehlen bzw. vorenthalten würden ${ }^{124}$.

${ }^{117}$ IORL, P\&S, 11, 259, 2500 (1925): Ameer Ali an FO, 10.9.1924.

${ }^{118}$ IORL, P\&S, 11, 259, 2500 (1925): FO an Ameer Ali, 18.9.1924.

${ }^{119}$ Moroccan War Sufferers, in: Times, 18.10.1924; The War in Morocco, in: Islamic Review 12 (1924) S. 412f,; ABBASI, London Muslim League, S. 380.

${ }^{120}$ A.S.M. ANIK, Correspondence (Leserbrief, 27.1.1925), in: Islamic Review 13 (1925)

S. 120; Riff Medical Relief Fund, in: Times, 26.11.1924.

${ }_{121}$ Telegrams in Brief, in: Times, 29.4.1925 und 27.5.1925.

${ }^{122}$ IORL, P\&J, 12, 230, 1671 (1924): Bericht „Extract from Report by New Scotland Yard, Money for Relief of Riffs«, 25.3.1925.

${ }^{123}$ CADN Tanger C40: Tabor de police $N^{\circ} 1$, Renseignements, 11.2.1926; ADMAE Maroc 133, 284: Tabor de police $\mathrm{N}^{\circ}$ 1, Renseignements, 21.3.1926.

${ }^{124} \mathrm{~S}$. Cyril B. MUNDEY, Correspondence (Leserbrief), in: Al-Moghreb al Aksa \& Tangier Gazette, 4.10.1924. 
Die Tangerer Helfer der British Red Crescent Society konnten in der Kürze der Zeit nicht mehr als einige kleine Pakete mit Medizin für Gordon-Cannings Mission auftreiben ${ }^{125}$. Der Apotheker Ernest Florentine Bonich stellte Medikamente und Verbandszeug zur Verfügung ${ }^{126}$. Bonich hatte 1903 eine Apotheke in der Tangerer Avenida de España mit einer weiteren Zweigstelle in Larache eröffnet und sich angesichts vermögender Stammkundschaft - wie zum Beispiel Ex-Sultan Abdelaziz - zu einem führenden Mitglied der englischen Gemeinschaft in Tanger emporgearbeitet ${ }^{127}$.

Ende Oktober 1924 brachen Gordon-Canning und sein Freund Gerald Spencer Pryse von Tanger aus mit einer Muli-Karawane und 50 Rif-Begleitern zu einer fünfwöchigen Reise auf ${ }^{128}$. Spencer Pryse hatte in London und Paris Kunst studiert und 1907 einen ersten Erfolg mit einer Ausstellung in Wien gefeiert. Während des Ersten Weltkrieges hatte der bekennende Sozialist vor seinen Militäreinsätzen unter anderem Poster für humanitäre Hilfsorganisationen gemalt ${ }^{129}$. Die Arzneien, die Gordon-Canning ins Rif brachte, dienten dazu, einen guten Eindruck zu hinterlassen, um so die eigentlichen Ziele seines Aufenthaltes zu unterstützen. Weitaus die meiste Zeit während der Gespräche ging es um politisch-wirtschaftliche Aspekte ${ }^{130}$. Gordon-Canning und Spencer Pryse erkannten, daß die Rifkabylen dringend medizinische Hilfe benötigten und versprachen baldige Hilfe ${ }^{131}$.

Im Rif fehlte es vor allen Dingen an ausgebildeten Ärzten. Mit dem gleichen Problem war die British Red Crescent Society in Tanger konfrontiert. Also entsandten Captain Mundey und seine Helfer Ende 1924 zwei Wagemutige, die sich als `Ärzte` auszugeben hatten, ins Rif. Der eine war ein schwarzer Drogist ${ }^{132}$ oder Krankenpfleger ${ }^{133}$ aus Tanger namens Mahboub. Bei Ajdir und

${ }^{125}$ PRO-FO 371, 11079, 39, 43: Gertrude Arnall an CG Tangier, 19.7.1925.

${ }^{126}$ ADMAE Maroc 135, 116: Tabor de police $\mathrm{N}^{\circ}$ 1, Information, 11.1.1926; PRO-FO 371, 12687, 11, 190: Ambassade de Londres an FO, 25.2.1927 mit beiliegendem Bericht "Annexe au memorandum présenté par le Gouvernement de sa Majesté«, 15.2.1927.

${ }^{127}$ PRO-FO 174, 303, 74, 9: CG Tangier an FO, 11.5.1924; VAIDON, Tangier, S. 180; WOOLMAN, Stars in the Firmament, S. 93.

${ }^{128}$ Two Englishmen among the Riff Tribesmen. Secret Mission to Abdel Krim, in: Manchester Guardian, 13.12.1924.

${ }^{129}$ Peter HARRINGTON, The Great War's Human Plight, in: MHQ, The Quarterly Journal of Military History 13 (2000) S. 48-53, S. 48f.; Who Was Who 5 (1962) S. 895f.

${ }^{130}$ SPENCER PRYSE, Through the Lines, S. 468-471. Siehe zu dieser Mission ebenfalls Kapitel 6, S. 319-321.

${ }^{131}$ HARRIS, Conditions in the Rif, 3.12.1924; Found Arabs Well Armed, in: New York Times, 3.12.1924.

${ }^{132}$ PenNell, A Country, S. 150, S. 154, Anm. 48; DerS., Women and Resistance to Colonialism in Morocco. The Rif 1916-1926, in: Journal of African History 28 (1987) S. 107118, S. 116. Fälschlicherweise gibt Pennell das Jahr 1921 als Beginn von Mahboubs Diensten im Rif an.

${ }^{133}$ SHAT 3H102: EMA, $2^{e}$ bureau, Bericht »Entourage d'Abd-el-Krim«, 25.8.1925; PARENT, Au Riff, S. 308. 
in Aïn el Halouf bei Morro Nuevo gründete er in der Folgezeit kleine Feldlazarette, wo er und einige von ihm ausgebildete Helfer mit ungenügenden Mitteln Verwundete und Kranke pflegten ${ }^{134}$. Nach dem spanischen Vormarsch im September 1925 wurden die Lazarette in Taghzout vereinigt und später nach Bouham verlegt. Die Methoden des Mahboub waren berüchtigt. Wunden und Blutungen rieb er - aus Mangel an Medikamenten - mit einer Mixtur aus Eiern und Jod ein ${ }^{135}$. Seine mangelnden Medizinkenntnisse offenbarte er bei vielen fehlgeschlagenen Operationen ${ }^{136}$. Kurz vor Kriegsende wurde das Feldhospital aufgelöst. Der unfähige Mahboub wurde zur Bewachung der Telefonverbindung zwischen Toufist und Bouham abkommandiert ${ }^{137}$.

Der andere von der British Red Crescent Society entsandte 'Arztı war der 1888 in Aachen geborene und 1903 in Norwegen eingebürgerte Walter Hüntjens ${ }^{138}$. Seit 1910 oder 1913 arbeitete er zusammen mit seiner Frau als Masseur in Tanger ${ }^{139}$. Die Arbeit brachte so wenig ein, daß Hüntjens Ende 1924 mit Captain Mundey einen Vertrag schloß, als Repräsentant der British Red Crescent Society ins Rif zu gehen, um die verwundeten Rifsoldaten zu ver-

${ }^{134}$ CADN Maroc DAI 189: Bericht "Notes sur une conversation tenue avec Abdelkrim le 31 mai 1926«; SHAT 3H102: Région d'Oujda, Contrôle civil de Taourirt, Bericht »Relation de voyage au Riff de Monsieur Courouble, correspondant du Quotidien«, 21.3.1925; KHARCHICH, La France, S. 113; Goded LloPIS, Marruecos, S. 90; PENNELL, A Country, S. 150.

${ }^{135}$ CADN Tanger C39: Tabor de police $\mathrm{N}^{\mathrm{o}}$ 1, Note, 18.9.1925; CADN Maroc DAI 192: RG Rabat an EMA, $2^{\mathrm{C}}$ bureau, 13.11.1925 mit dem Bericht »Renseignements fournis par le sergent-fourrier Cucchi« o.D.; CADN Tanger C38: CG Tanger an RG Rabat, 3.3.1926.

${ }^{136}$ LANGLET, Riff, S. 180f.; ADMAE Maroc 134, 57: Ambassade de Stockholm an MAE, 5.5.1926 mit Auszügen aus Hans Langlets Artikel in der »Dagens Nyheter《, 25.4.1926; CADN Maroc DAI 192: Bericht "Rapport du Docteur Mosnier «, 1.6.1926.

${ }^{137}$ PARENT, Au Riff, S. 94. Mahboub starb in den 1970ern in Tanger [PENNELL, A Country, S. 154, Anm. 48].

${ }^{138}$ PA-AA Madrid 138: Konsulat Tetuan an Botschaft Madrid, 4.7.1927. Hüntjens' Name taucht in Quellen und in der Literatur in mannigfaltigen Variationen auf: "Walter Heintgent" [WOOLMAN, Rebels in the Rif, S. 151]; "Huntgens Klein « [CADN Tanger C39: Tabor de police $\mathrm{N}^{\circ}$ 1, Information, 17.7.1925]; »Hintchkins « [CADN Maroc DAI 199: EMA, $2^{c}$ bureau an RG Rabat, 6.7.1926]; »Hunzrus« [IORL, P\&J, 12, 230, 1671 (1924): A. Merry del Val an Lord Birkenhead, 18.3.1925]; "Walter Hutyens" [HARRIS, France, S. 225; PENNELL, A Country, S. 177, S. 192, Anm. 4]; »Hinz« [GABRIELLI, Abd-el-Krim, S. 180]; »Hudgeus Klein« [OVED, La gauche française, Bd. 1, S. 294; DERS., La contribution des services spéciaux à la politique de la France au Maroc de 1920 à 1955, in: Claude JourNès (Hg.), Police et politique, Lyon 1988, S. 69-99, S. 96, Anm. 21]. Es handelt sich jedoch zweifelsfrei um dieselbe Person.

${ }^{139}$ SHAT 3H1558: Tabor de police $\mathrm{N}^{\circ} 1$, Note, 13.3.1925; CADN Tanger C39: Tabor de police $\mathrm{N}^{\circ}$ 1, Information, 17.7.1925; PA-AA Madrid 138: Konsulat Tetuan an Botschaft Madrid, 4.7.1925. 
sorgen. Jeden Monat überwies Mundey der Frau von Walter Hüntjens, die im Tangerer Hôtel des sceurs latines - später im Hôtel de Paris - wohnte, den verabredeten Geldbetrag ${ }^{140}$.

In Chichaouen hatten die spanischen Truppen nach ihrem Rückzug nach Tetuan Ende 1924 ein kleines Hospital mit einem Operationsraum und drei Stationen zu je acht Betten hinterlassen. Im falschen Glauben, Hüntjens habe ausgereifte medizinische Kenntnisse, übertrug ihm Abdelkrim die Aufgabe, die Leitung des Hospitals zu übernehmen. Dem amerikanischen Journalisten Vincent Sheean zufolge, der das Rif im Januar 1925 aufsuchte, vermittelte Hüntjens sogar den Eindruck eines fähigen Chirurgen. Ihm zur Seite standen von Si M'hammed zur Verfügung gestellte Einheimische ${ }^{141}$. Schnell kam heraus, daß das Vertrauen der Rifkabylen in Hüntjens' angebliche Fähigkeiten ungerechtfertigt war. Abdelkrim setzte ihn Ende Februar 1925 als Leiter des Hospitals $a b^{142}$. Hüntjens wurde nunmehr als Topograph und Leiter des Straßenbaus eingesetzt ${ }^{143}$. In der Folgezeit übernahmen die Rifkabylen die Organisation des Sanitätswesens selbst ${ }^{144}$. Im Sommer 1925 bezeichnete sich Caïd Haddou gegenüber dem amerikanischen Journalisten Larry Rue als „Oberbeauftragten für das Gesundheitswesen « ${ }^{145}$. Mehr als rudimentäre Strukturen kamen aber nie zustande.

Unterdessen bemühten sich die Rif-Sympathisanten in London weiter darum, von der britischen Regierung die offizielle Unterstützung für eine humanitäre Hilfsmission zu erhalten. Nachdem die Near and Middle East Association durch ihren Geschäftsführer Ernest Nathaniel Bennett die seit Juli 1924 unterbrochenen Aktivitäten wieder aufgenommen und am 25. November 1924 in der Londoner "Times" und im "Manchester Guardian« einen flammenden Appell an die britische Hilfsbereitschaft veröffentlicht hatte ${ }^{146}$, richtete sich die nächste Initiative direkt an das Außenministerium. In seinem Schreiben vom 19. Dezember 1924 bat Bennett um Beistand für die Entsendung von Hilfsgütern. Sowohl die Zivilbevölkenung als auch die Kombattanten im Rif würden schrecklich unter dem Mangel an Arzneien leiden, schrieb Bennett. Er

${ }^{140}$ PRO-FO 371, 12687, 11, 190: Ambassade de Londres an FO, 25.2.1927 mit beiliegendem Bericht "Annexe au memorandum présenté par le gouvernement de sa Majesté«, 15.2.1927; CADN Tanger C39: Tabor de police $\mathrm{N}^{\circ}$ 1, Information, 17.7.1925.

${ }^{141}$ SHEEAN, An American, S. 250f.; DERS., »Robin Hood« of the Riff. The »German Pilgrim«, Aid to Abd el-Krim and to the Riffi, »Great Man«, His Brother, in: Asia 25 (1925) S. 969-980, S. 977. Fälschlicherweise ging Sheean davon aus, daß der Leiter des Hospitals ein Däne war. Vgl. PENNELl, A Country, S. 177, S. 192, Anm. 4; Youssoufi, Les institutions, S. 98.

${ }^{142}$ CADN Tanger C39: Tabor de police $\mathrm{N}^{\circ} 1$, Information, 17.7.1925 und 8.8.1925.

${ }^{143}$ Siehe hierzu und zu Hüntjens' weiterem Schicksal Kapitel 4, S. $128 \mathrm{f}$.

${ }^{144}$ USBORNE, The Conquest, S. 270.

${ }^{145}$ RUE, News, S. 273.

${ }^{146}$ TOYNBEE, The Islamic World, S. 123, Anm. 6; Medical Supplies for the Rifi, in: Manchester Guardian, 25.11.1924. 
fügte warnend hinzu, daß das Embargo für derartige Lieferungen in der moslemischen Welt, darunter auch Indien, große Entrüstung hervorrufe ${ }^{147}$. Das Außenministerium lehnte - wie schon zuvor - jegliche Unterstützung ab und verwies Bennett an die spanische Regierung ${ }^{148}$. In einem ausführlichen Leserbrief an den »Manchester Guardian« beklagte sich Bennett über diese Haltung und brachte seine Bestürzung darüber zum Ausdruck, da $B$ es hingenommen werde, Rebellen die Hilfe, auf die jeder Mörder in europäischen Ländern Anspruch habe, zu verweigern ${ }^{149}$.

Die British Red Crescent Society bereitete derweil wegen alarmierender Nachrichten aus dem Rif eine weitere Hilfsmission in Eigenregie vor. Diesmal sollten umfangreichere Hilfsgüter sowie ein englischer und ein moslemischer Krankenpfleger zu Abdelkrim gesandt werden ${ }^{150}$. Gerald Spencer Pryse bemühte sich, für den Transport ein Schiff aufzutreiben ${ }^{151}$. Dr. Ernest Griffin war für die Leitung der Mission vorgesehen. Der Arzt ignorierte die seit Jahren ablehnende Haltung der britischen Regierung völlig. Anfang Januar 1925 bat er um die Ausstellung einer Exportgenehmigung für Arzneien. Er fügte hinzu, daß er beabsichtige, die Medikamente Abdelkrim persönlich zu übergeben, und er gab einen naiven Einblick in seine Reisepläne: "Vertraulich. Ich plane, in Marseille verladen zu lassen und von dort ins Rif überzusetzen. Wären Sie so gut, mich wissen zu lassen, wo ich die erforderliche Genehmigung einholen kann? ${ }^{152}$ Das Innenministerium teilte Griffin am 15. Januar 1925 daher bestimmt mit, daß die Exportbewilligung ohne die Erlaubnis der zuständigen französischen und spanischen Behörden nicht erteilt werde ${ }^{153}$. Das bedeutete das Ende für Griffins Planungen. So mußte Gerald Spencer Pryse später in einem Brief an Abdelkrim einräumen, daß das Schiff mit dem versprochenen Sanitätsmaterial nicht gekommen sei, weil Spanien die Entsendung nicht erlaubt habe ${ }^{154}$.

Lediglich aus Algerien gab es noch sporadische Hilfe für die Rifkabylen. Bereits im Oktober 1924 hatte der ausgebildete Sanitäter Raymond Autun, dessen Vater als Arzt im algerischen Mers el Kebir arbeitete, Medikamente im Wert von 5000 Francs ins Rif geschickt. Anfang Dezember 1924 versuchte er, selbst dorthin zu gelangen, um die Verwundeten zu pflegen. Diesmal hatte er 1200 Francs für Arzneien investiert. Autun kaufte ein Motorboot, das jedoch

\footnotetext{
${ }^{147}$ PRO-FO 371, 10586, 7382, 17: E.N. Bennett an FO, 19.12.1924.

${ }^{148}$ IORL, P\&S, 11, 259, 2500 (1925): FO an E.N. Bennett, 29.12.1924.

149 Ernest N. BENNETT, Correspondence. The Red Cross and the Riff, in: Manchester Guardian, 23.1.1925.

${ }^{150}$ The Medical Mission to the Riffs, in: Manchester Guardian, 23.12.1924.

${ }^{151}$ PRO-FO 371, 11080, 94, 10: Embajada de Londres an FO, 31.1.1925.

${ }^{152}$ PRO-FO 371, 11081, 280, 2: HO an FO, 8.1.1925 mit beigefügtem Brief Ernest H. Griffin an HO, 5.1.1925.

${ }^{153}$ PRO-FO 371, 11081, 240, 57: HO an Ernest H. Griffin, 15.1.1925.

154 CADN Maroc DAI 198: Gerald Spencer Pryse an Abdelkrim, 28.9.1925.
} 
am 9. Dezember 1924 von der französischen Küstenwache aufgebracht wur$\mathrm{de}^{155}$. Drei Tage später gelang es ihm, in Begleitung des Journalisten Jacques de Marsillac von der französischen Zeitung "Journal« über Taourirt und Guercif das Rif zu erreichen. Hier verliert sich seine Spur ${ }^{156}$. Der Algerienfranzose Athias aus Oujda schickte Ende Januar 1925 Äther, Alkohol, und Medikamente ${ }^{157}$.

Spärliche Unterstützung kam aus den nordafrikanischen Nachbarländern. Ein Comité d'assistance pour les victimes du Rif aus Kairo übermittelte der französischen Botschaft am 8. März 1925 die Bitte, eine humanitäre Hilfsmission zuzulassen ${ }^{158}$. Prinz Omar Toussoun hatte bereits 1923 in Kairo das Comité du secours aux sinistrés rifains gegründet. Er bezahlte 1925 Frachtlieferungen der Jacht »Pharaon ${ }^{159}$. Im Sommer 1925 hielten sich ägyptische und tunesische Ärzte im Rif auf ${ }^{160}$. Derartige Aktionen hielten sich aber sehr in Grenzen und waren weniger als ein Tropfen auf den heißen Stein.

Anfang 1925 suchte Abdelkrim über seinen Tangerer "Briefkasten« Gertrude Arnall den Kontakt nach Indien. Arthur Field hatte von dem 1919 aus Indien ausgewiesenen Journalisten Benjamin Guy Horniman den Ratschlag erhalten, von indischen Moslems Geldspenden für das Rif einzusammeln ${ }^{161}$. Horniman hatte 1913 den "Bombay Chronicle« gegründet und war in der Folgezeit zum Präsidenten der Press Association of India gewählt worden. Vor seiner Ausweisung aus Indien hatte er kritische Artikel über Massaker britischer Truppen bei Ausschreitungen im Punjab veröffentlicht ${ }^{162} .1920$ publizierte Horniman hierüber zwei Hefte (»Amritsar and our Duty to India«, »The Agony of Amritsar and the Reign of Terror in the Punjab«). Seither versuchte er vergeblich, seine Arbeit in Indien wiederaufnehmen zu können. Eine Petition vom 21. Juli 1924 forderte die Erlaubnis für Hornimans Rückkehr nach

155 ADMAE Maroc 219, 164: Rapport du chef de la sûreté départementale Oran, 12.12.1924; CADN Maroc DAI 197: Région d'Oujda, Renseignements, 20.12.1924.

${ }^{156}$ SHAT 3H1557: Contrôle civil de Taourirt, Bulletin de renseignements, 15.12.1924; ADMAE Maroc 219, 183: Sous-Préfet de Tlemcen an Préfet d'Oran, 26.12.1924.

${ }^{157}$ SHAT 3H1561: Commandement supérieur des troupes du Maroc, EMA, $2^{e}$ bureau, Bericht »Renseignements divers foumis par Antonio Palma«, 1.1.1926.

${ }^{158}$ CADN Caire 103: Comité d'assistance pour les victimes du Rif an Ambassade de Caire, 8.3.1925.

${ }^{159}$ CADN Londres C536: Ministère des colonies, Bericht »Revue de la presse et des questions musulmans«, 15.10.1923; AN F7, 13413: Bericht »L'aide aux Riffains et les relations avec Abd el Krim«, 12.11.1925; CADN Madrid C263: Ambassade de Madrid an MAE, 30.7.1925 und 19.8.1925; CADN Tanger C38: RG Rabat an CG Tanger, 4.3.1926.

${ }^{160}$ SHAT 3H102: Bericht »Entourage d'Abd-el-Krim«, 25.8.1925; L'agression des Rifains contre le Maroc français, in: BCAF 35 (1925) S. 251-262, S. 259.

${ }^{161}$ PRO-FO 371, 11079, 39, 70: New Scotland Yard an FO, 10.8.1925; AN F7, 13413: MAE an MI, Affaires indigènes, 26.8.1925.

${ }_{162}$ Mr. B.G. Horniman, in: Times, 18.10.1948; Who's Who 78 (1926) S. 1432; Who Was Who 4 (1952) S. 564; PRO-FO 372, 2092, 1470, 124: IO an FO, 5.3.1924. 
Indien. Sechs Unterzeichner gehörten dem ein Jahr später in London gegründeten Riff-Committee an: Lt.-Cdr. Joseph Montague Kenworthy (Liberal Party), F. de Vere Summers (National Liberal Club), Khalid Sheldrake (EastWest Circle), Ernest H. Griffin, Ronald G. Armstrong und Arthur Field ${ }^{163}$. Es ist denkbar, daß Horniman in Indien für die Rifsache agitieren sollte. Er war klar antiimperialistisch eingestellt und hoffte, wie er am 30. Januar 1922 auf einer Konferenz in London ausgeführt hatte, daß der Erfolg der britischen Arbeiterbewegung unausweichlich andere Völker von der Kontrolle fremder Imperialisten und Kapitalisten befreien würde ${ }^{164}$. Erst im Januar 1926 allerdings sollte Horniman über Ceylon die Rückkehr nach Indien gelingen ${ }^{165}$.

Anfang 1925 reisten zwei Gesandte nach Indien, um für die Sache der Rifkabylen zu werben. Im Februar 1925 traf sich der Marokkaner Mohammed ben Abdelkader Halali in Bombay mit Moslems aus Nordindien. Sie wurden von Dr. Mohammed Naïm angeführt, der in Lucknow (heute Lakhnau) bereits mit den Vorbereitungen für eine Hilfsmission begonnen hatte. Der Rifgesandte bereiste von Februar bis März 1925 weite Teile Indiens, um Propaganda für Abdelkrim zu betreiben. Der Ägypter Scheich Mohammed Faraj, der Präsident der Association of Learned Men in Kairo, sollte als Vermittler zwischen Naïm und Abdelkrim fungieren ${ }^{166}$. Zwar wurden Medikamente gesammelt, doch weder die Arzneien noch die geplante Hilfsmission kamen jemals im Rif an ${ }^{167}$.

Der zweite Indien-Gesandte war der Türke Mohammed ben Saadedine Djibaoui. Der Tangerer Rif-Agent Moulay Ali el Khamlichi hatte ihn im Frühjahr 1925 bei Abdelkrim eingeführt. Gute Kontakte hatte er auch zu Gertrude Arnall. Ein Portrait-Foto von ihm hing in der Vitrine ihres Tangerer Fotoateliers. Über Gertrude Amalls Londoner Freunde Arthur Field und Benjamin G. Horniman wurde für ihn die Verbindung nach Indien hergestellt ${ }^{168}$. Anfang Mai 1925 traf sich Djibaoui in Bombay mit Vertretern des Punjab Khilafat Committees. Er machte deutlich, daß das Rif weniger Geld und MoslemKämpfer, sondern eher Medikamente und ärztlichen Beistand benötige. Auch diese Mission brachte keinen Erfolg. Das Punjab Khilafat Committee war nur

${ }^{163}$ PRO-FO 372, 2092, 1470, 143: Arthur Field an Ronald Waterhouse, Secretary to the Prime Minister, 21.7.1924.

${ }^{164}$ Mesbahuddin AHMED, The British Labour Party and the Indian Independence Movement 1917-1939, London 1987, S. 49.

${ }^{165}$ PRO-FO 372, 2245, 596, 129: IO an FO, 13.1.1926.

166 IORL, P\&S, 11, 259, 2500 (1925): Consul général de Kalkutta an Deputy Secretary to the Government of India, Dehli, 27.3.1925 und 8.5.1925, Deputy Secretary to the Government of India, Dehli an Consul général de Kalkutta, 29.4.1925.

${ }^{167}$ PENNELL, A Country, S. 150; LADREIT DE LACHARRIERE, Le rêve, S. 155.

${ }^{168}$ SHAT 3H102: EMA, $2^{e}$ bureau, Bericht "Contrebande à Tanger«, 25.7.1925. 
zu einer Geldüberweisung über die Imperial Bank of India bereit. Da es keinerlei Möglichkeiten gab, das Geld ins Rif zu transferieren, wurde es dem Hedjaz Relief Fund gespendet ${ }^{169}$.

Hartnäckig hielten sich nach dem Angriff der Rifkabylen auf die französische Zone Marokkos Gerüchte, daß Deutschland Abdelkrim Militärärzte und Hebammen zu Hilfe geschickt habe ${ }^{170}$. Hierfür gibt es keinerlei Anhaltspunkte. Vereinzelte Hilfsangebote gab es aber tatsächlich. In Marrakesch fingen die französischen Behörden einen vom 24. Juli 1925 datierten Brief aus München ab. Der wissenschaftliche Leiter der Pantosept GmbH Chemische Fabrik Ehrenstein Max Clasz fügte seinem Schreiben wan S.M. Abd el Krim, Kriegsschauplatz Marokko« eine Verkaufsliste mit Verbandsmaterial und antiseptischen Mitteln bei ${ }^{171}$. Der 34jährige deutsche Arzt Joseph Raimann aus Prag schrieb Abdelkrim am 1. August 1925 auf die Postkarte: „Möchte (mich) mit Ihren Truppen am Kampfe gegen Frankreich beteiligen ${ }^{172}$. Und ein Dr. med. A. Kummer aus Breslau fragte im Auswärtigen Amt nach, ob man dort die Adresse der »Medizinal-Abteilung der Rifleute« kenne, da er seine »ärztlichen Kräfte den Rifkabylen in Afrika anzubieten « gedachte ${ }^{173}$. Diese Angebote blieben Einzelfälle. Niemand schaffte den Weg nach Marokko.

Im Rif herrschte im Sommer 1925 wegen der zusätzlichen Kämpfe gegen französische Truppen und wegen einer Typhusepidemie akuter Medikamentenmangel ${ }^{174}$. Seit August 1925 bemühte sich die British Red Crescent Society ein weiteres Mal um die Entsendung einer Hilfsmission ins Rif. In diesem Fall wandte sich Syed Ameer Ali nicht an seine eigene, sondern direkt an die französische Regierung. In seinem Schreiben an die französische Botschaft in London vom 30. September 1925 bemühte er sich um moderate Töne. Er hob hervor, daß die Zustimmung für eine Hilfsmission - erneut geplant unter der Führung von Dr. Ernest Griffin - Frankreichs Ansehen in der moslemischen Welt anheben würde ${ }^{175}$. Der Vorstoß blieb unbeantwortet. Syed Ameer Ali gab jedoch nicht auf. Am 17. Dezember 1925 schrieb er das französische AuBenministerium ein weiteres Mal mit der Bitte an, eine Mission ins Rif entsen-

${ }^{169}$ IORL, P\&S, 11, 259, 2500 (1925): Deputy Secretary to the Government of India, Dehli an Consul général de Kalkutta, 13.8.1925; What the Riffians Need, in: Muslim Outlook, 7.5.1925.

${ }^{170} 540$,touristes allemands sont arrivés à Tétouan, in: Intransigeant, 26.5.1925; Des officiers allemands auprès d'Abd-el-Krim, in: Homme Libre, 1.6.1925; PA-AA Botschaft Paris 454a: Botschaft Paris an AA, 4.6.1925.

${ }^{171}$ CADN Maroc DAI 196: Région de Marrakèch an RG Rabat, 6.8.1925.

17 CADN Maroc DAI 199: Postkarte im Ordner "Mappe saisie de la correspondance d'Abdelkrim transitant par la zone françaiser mit grammatikalischer Korrektur.

${ }_{173}$ PA-AA R30190k: Dr. U. Kummer an AA, 15.9.1925, AA an Dr. Kummer, 23.9.1925.

${ }^{174}$ ABDELKRIM, Memoiren, S. 95, S, 145; PORCH, Foreign Legion, S. 405.

${ }^{175}$ CADN Londres C527: Ameer Ali an Embassy of France, 30.9.1925. 
den zu dürfen ${ }^{176}$. Die neue Regierung unter Ministerpräsident Aristide Briand hatte jedoch an Friedensinitiativen oder sonstigen Einmischungen kein Interesse mehr, da Abdelkrim militärisch so gut wie geschlagen war. Eine Replik an Ameer Ali blieb aus. Genauso blieben dessen Petitionen vom 6. März und vom 8. Juni 1926 unbeantwortet ${ }^{177}$.

Im Verlaufe des Jahres 1925 war der Kontakt des IKRK nach Spanien nie abgerissen. Das spanische Rote Kreuz hatte Nachforschungsbüros für vermißte deutsche und portugiesische Soldaten der spanischen Fremdenlegion eingerichtet. Nach wie vor erhielten die Rifkabylen hingegen keinerlei Hilfe. Das IKRK kümmerte sich nun auch um das Schicksal der Kriegsgefangenen innerhalb des Rifs, denen es ebenfalls an medizinischer Versorgung mangelte. Gustave Ador, der schweizerische Präsident des IKRK, nahm die 6. Generalversammlung des Völkerbundes im September 1925 als Gelegenheit für ein direktes Gespräch mit dem damaligen französischen Ministerpräsidenten Paul Painlevé und dessen Außenminister Aristide Briand wahr. Raymond Schlemmer debattierte zusätzlich mit dem spanischen Botschafter von Paris Quiñones de Léon sowie mit dessen Berner Kollegen Palacios de Fau. Briand versprach, den französischen Generalstab mit der Planung eines Hilfsprojektes zu beauftragen. Da das IKRK bis Mitte Oktober 1925 noch keinerlei Nachricht erhalten hatte, plante es bereits, auf eigene Faust eine Mission unter Führung von Dr. F. Blanchod nach Marokko zu entsenden. Die Hoffnungen, daß Frankreich diese Mission dulden würde, zerstoben, als der französische Generalkonsul von Genf dem IKRK am 26. Oktober 1925 einen Brief Briands übermittelte. Darin hieß es, die kombinierte spanisch-französische Militäraktion lasse keine sofortige Lösung betreffend der Anfrage des IKRK zu. Da die militärischen Einsätze wegen des hereinbrechenden Winters für 1925 beendet seien, wurde eine Hilfsmission als unnötig betrachtet. Den wenigen französischen Gefangenen im Rif, fügte Briand hinzu, komme bereits Hilfe $\mathrm{zu}^{178}$.

${ }^{176}$ CADN Madrid C263: British Red Crescent Society an MAE, 17.12.1925; ABBASI, London Muslim League, S. 379-381.

${ }^{177}$ ADMAE Maroc 135, 89: Section d'étude de la presse étrangère, 4.1.1926 (Leserbrief von Ameer Ali in der Times, 4.1.1926, mit seiner Beschwerde, keine Antwort von der französischen Regierung erhalten zu haben); CADN Londres C520: Ameer Ali an Embassy of France, 6.3.1926 und 8.6.1926.

${ }^{178}$ DuRAND, Histoire, Bd. 2, S. 198f;; La Croix Rouge et le Rif, in: BISCR 56 (1925) S. 945f.; CADN Madrid C261: MAE an Ambassade de Madrid, 29.10.1925. Kein Wort über Gustave Adors Rifkrieg-Initiative enthält der anläßlich seines 150. Geburtstages (1995) herausgegebene Sammelband von Roger Durand, Daniel Barbey und Jean-Daniel Candaux [Roger DuRAND, Daniel BARBEY, Jean-Daniel CANDAUX (Hg.), Gustave Ador. 58 ans d'engagement politique et humanitaire. Actes du colloque Gustave Ador tenu au palais de l'Aténée les 9, 10 et 11 novembre 1995, Genf 1996] oder die Biographie Frédéric Barbeys [Frédéric BARBEY, Un homme d'état suisse. Gustave Ador 1845-1928, Genf 1945]. 
Bis zum Frühjahr 1926 lag dem IKRK kein offizielles Hilfsgesuch von Abdelkrim selbst vor - vermutlich aus Unkenntnis. Dies hätte die Genfer bindend zu Aktivitäten verpflichtet ${ }^{179}$. Der schwedische Journalist Hans Alexander Langlet und sein Freund Waldemar Lanke hatten über die Vermittlung von Captain Robert Gordon-Canning vom 19. Januar bis zum 28. März 1926 das Rif bereist ${ }^{180}$. Zurück in Schweden übergab Langlet Prinz Karl, dem Präsidenten des schwedischen Roten Kreuzes, einen vom 21. März 1926 datierten Brief von Abdelkrim, in dem dieser im Namen der Humanität um medizinische Hilfe bat ${ }^{181}$. In dem Schreiben beklagte sich Abdelkrim bitter über die bisherige Weigerung seiner Kriegsgegner, Hilfslieferungen zuzulassen: "Wer ist grausamer als derjenige, der sich weigert, Schwache und Unglückliche zu versorgen? Sie [Spanien und Frankreich] haben es nicht zugelassen, daß unsere Verletzten Versorgungsgüter erhielten, um ihre Leiden zu lindern. Fürwahr, ein derartiger Akt ist unmenschlich ${ }^{182}$.

In einem Artikel der »Dagens Nyheter « vom 25. April 1926 forderte Langlet das Rote Kreuz eindringlich zu Hilfsmaßnahmen für die Rifkabylen auf, Auf Anraten des schwedischen Außenministeriums weigerte sich Prinz Karl zwar, Langlet persönlich zu empfangen, aber er leitete Abdelkrims Brief am 30. April 1926 mit der Bitte an das IKRK weiter, entsprechende Maßnahmen zu ergreifen ${ }^{183}$. Zum einen lag dem IKRK nun ein direkter Appell Abdelkrims vor, der erwarten ließ, daß eine Hilfsmission im Rif willkommen sein würde. Zum anderen konnte das IKRK davon ausgehen, auf die Hilfe einer wichtigen nationalen Rotkreuz-Gesellschaft zählen zu können. Der sofort entsandte Raymond Schlemmer traf am 8. Mai 1926 in Rabat ein. Aber er kam zu spät. Am selben Tag war eine französische Hilfsmission ins Rif aufgebrochen. Sie galt den alliierten Kriegsgefangenen. Als Schlemmer am 25. Mai 1926 von der französischen Generalresidenz die Erlaubnis erhielt, diese Hilfsmission zu unterstützen, endete der Krieg zwei Tage später mit der Kapitulation Abdelkrims $^{184}$.

${ }^{179}$ La Croix Rouge et le Rif, in: BISCR 56 (1925) S. 947.

${ }^{180}$ LANGLET, Riff, S. 39, S. 87; ADMAE Maroc 134, 98: Bericht »Note au sujet du suédois Alexandre Langlet«, 19.4.1926; ADMAE Maroc 134, 20: Ambassade de Stockholm an MAE, 8.4.1926.

${ }^{181}$ DURAND, Histoire, Bd. 2, S. 200; DaOUD, Abdelkrim, S. 324; Le typhus décimerait les Rifains? Abd el Krim demanderait aide sanitaire, in: Petit Journal, 9.4.1926.

${ }^{182}$ Zit. aus: Youssoufl, Les institutions, S. 98.

${ }^{183}$ ADMAE Maroc 134, 53: Ambassade de Stockholm an MAE, 4.5.1926 und 5.5.1926.

${ }^{184}$ RIESENBERGER, Für Humanität, S. 53; DURAND, Histoire, Bd. 2, S. 200f.; BUGNION, Le Comité, S. 338. 


\subsection{Die Rif-Flüchtlinge in Tanger und der Refugee Relief Fund}

Von Mai bis Juni 1925 grassierte in der internationalen Zone von Tanger eine Pockenepidemie, die die dort vorherrschende Flüchtlingsproblematik noch verschärfte. 1517 Impfungen mußten vorgenommen werden ${ }^{185}$. Etwa 5000 bis 7000 Männer, Frauen und Kinder waren bisher vor den Kämpfen im Rif ins vermeintlich sichere Tanger geflohen. Der im März 1924 von Lady Alice Drummond Hay, Feridah Kirby Green und vom kanadischen Pastor H.P. Elson gegründete Refugee Relief Fund trat der Epidemie und der drohenden Hungersnot entgegen ${ }^{186}$. Lady Drummond Hay war die Tochter des berühmten Tangerer Generalkonsuls John Drummond Hay. Sie kannte die Verhältnisse im Rif aus eigener Anschauung und fungierte nun als Korrespondentin des Londoner "Daily Express ${ }^{187}$. 1866 war Feridah Kirby Green als vierzehnjährige Diplomatentochter nach Tanger gekommen. Die Kirby Greens gehörten daher in den 1920er Jahren bereits zu den alteingesessenen englischen Familien. Noch mit 80 Jahren verteilte Feridah Kirby Green einmal pro Woche Geschenke an arme marokkanische Frauen ${ }^{188}$.

Die finanziellen Mittel der Helfer erschöpften sich trotz der wöchentlichen Unterstützung der British Red Crescent Society in Form von 50 Pfund rasch. In einem Appell in der "Times« vom 22. Juni 1925 bat Syed Ameer Ali die britische Öffentlichkeit um Spenden ${ }^{189}$. Drei Tage später wandte er sich eindringlich an das britische Außenministerium. Er beklagte, daß die neue Verwaltung der internationalen Zone das Gesundheitswesen bisher vernachlässigt habe. Da Großbritannien als eine von drei Mächten an der Tanger-Administration beteiligt sei, verpflichte dies die britische Regierung, Bedürtigen zu helfen. Ameer Ali bat um finanzielle Unterstützung, damit die British Red Crescent Society den Flüchtlingen in Tanger helfen könne ${ }^{190}$. Jedoch wurde jegliche Verantwortung mit der Begründung abgelehnt, daß Tanger selbst für die Versorgung der Flüchtlinge zuständig sei ${ }^{191}$.

${ }^{185}$ La Croix-Rouge espagnole à Tanger, in: BISCR 57 (1926) S. 208f., S. 208.

${ }^{186}$ Correspondence, in: Al-Moghreb al Aksa \& Tangier Gazette, 10.4.1926; CADN Tanger C37: CG Tanger an RG Rabat, 16.9.1925.

${ }^{187}$ Ein unbesiegtes Volk, in: Deutsches Offiziersblatt 30 (1925) S. 307f., S. 307; Nach der Einnahme von Aschdir, in: Berliner Tageblatt, 4.10.1925.

${ }^{188}$ Rom LANDAU, Portrait of Tangier, London 1952, S. 144, S. 148; WOOLMAN, Stars in the Firmament, S. 143; VADON, Tangier, S. 282f.

${ }^{189}$ ABBASI, London Muslim League, S. 379f.; PRO-FO 371, 11075, 18, 71: CG Tangier an FO, 7.8.1925.

${ }^{190}$ PRO-FO 371, 11075, 18, 74: Ameer Ali an FO, 25.6.1925.

${ }^{191}$ PRO-FO 371, 11074, 18, 40: Parliamentary Question, 18.6.1925; PRO-FO 371, 11075, 18, 77: FO an Ameer Ali, 8.7.1925. 
Was die Diplomaten tatsächlich dachten, verdeutlicht eine Notiz vom 29. Juni 1925: "Die neue Verwaltung verfügt über ungenügende Geldmittel für die Hilfe. So sehr wir jedoch auch mit den Flüchtlingen sympathisieren, können wir nicht zulassen, daß der britische Steuerzahler die Kosten für Hilfsmaßnahmen trägt ${ }^{192}$. Der erboste Lord Lamington von der British Red Crescent Society erinnerte daraufhin während einer Sitzung des britischen Oberhauses am 28. Juli 1925 daran, daß Großbritannien an der Aufteilung Marokkos, die das Flüchtlingsproblem verursacht habe, vertraglich beteiligt gewesen sei $^{193}$.

Spenden kamen insbesondere von persönlichen Freunden des Pfarrers Elson sowie aus der englischen Kolonie Tangers. Als Lady Drummond Hay Mitte Juni $1925 \mathrm{zu}$ ihrem jährlichen Sommerurlaub nach Großbritannien aufbrach, übernahm Elson die Leitung des Flüchtlingswerkes in Tanger. Die internationalen Behörden hatten die Ausgabe von Essensrationen innerhalb der Stadtgrenzen verboten. Zweimal pro Woche - mittwochs und freitags - organisierte Elson daher auf einem eigens dafür gekauften Stück Land zwei Meilen westlich außerhalb der Stadt (Djema el Mokra) die Verteilung von Mehl. Hierfür standen jeweils 20 bis 25 Hundert-Kilogramm-Säcke zur Verfügung. $\mathrm{Zu}$ den Ausgabetagen kamen bis zu 1500 Menschen. Nicht jeder hatte das Glück, etwas zu erhalten. Ein Kontrollsystem mit Essenskarten sollte Mehrfachverteilungen oder Mißbräuche ausschließen ${ }^{194}$. In einem Brief an Syed Ameer Ali beschrieb Elson die dramatischen Ereignisse im Flüchtlingscamp. Hungernde versuchten sich vorzudrängeln, Kinder litten an Pocken, Frauen mit Schußwunden mußten versorgt werden, Babys starben in den Armen ihrer Mütter ${ }^{195}$.

Schließlich sprangen die englischen Quäker ein. Die Society of Friends entsandte Ende Juli 1925 eine zweiköpfige Delegation nach Tanger, um die Lage zu sondieren ${ }^{196}$. Es handelte sich um den 63jährigen englischen Politiker Sir Charles Edward Henry Hobhouse und um die Friedensaktivistin Anna Ruth Fry. Hobhouses politische Laufbahn hatte 1922 in Birmingham ein Ende gefunden, als seine Kandidatur für die Unterhauswahlen gescheitert war. Als liberaler Abgeordneter hatte er von 1885 bis 1895 und von 1900 bis 1918 dem britischen Unterhaus angehört und seine Karriere als Parlamentssekretär für Indien (1907-1908) und als Staatssekretär im Finanzministerium (1908-1911)

\footnotetext{
${ }^{192}$ PRO-FO 371, 11075, 18, 72: Minute, 29.6.1925.

${ }^{193}$ Refugees in Tangier, in: Times, 29.7.1925 und 8.8.1925.

${ }^{194}$ Henri MENTHA, Mission à Tanger, in: BISCR 56 (1925) S. 971-981, S. 977-980; Relieving Refugees from the Riff, in: Al-Moghreb al Aksa \& Tangier Gazette, 19.9.1925; PROFO 371, 11075, 18, 71: CG Tangier an FO, 7.8.1925.

${ }^{195}$ PRO-FO 371, 11075, 18, 24: Ameer Ali an FO, 29.7.1925 mit beigefügtem Brief H.P. Elson an Ameer Ali, 23.7.1925.

${ }^{196}$ PRO-FO 371, 11083, 7118, 95: Ameer Ali an FO, 28.7.1925.
} 
gekrönt ${ }^{197}$. Ruth Fry, die Tochter des englischen Diplomaten Sir Edward Fry, entstammte einer alten Quäkerfamilie und hatte als Generalsekretärin des Friends War Victims Relief Committee (1914-1924), das den Opfern des Ersten Weltkrieges - auch im Ruhrgebiet - Hilfe zukommen lieB, und als Vorsitzende des Russian Famine Relief Fund (1921) bereits Erfahrungen in Katastrophengebieten gesammelt ${ }^{198}$.

Gleich nach ihrer Ankunft in Tanger trafen sich Hobhouse und Fry mit dem britischen Generalkonsul Robert Clive. Dieser hoffte, daß sich die Quäker anstelle der britischen Regierung finanziell für die Flüchtlinge engagieren würden. Die politische Bedeutsamkeit der Hilfsmaßnahmen sollte so gering wie möglich gehalten werden ${ }^{199}$. Hobhouse und Fry wurde klar, daß von ihrer Regierung keine Hilfe zu erwarten war ${ }^{200}$. Ruth Fry machte daher in einem Artikel in der von der Union of Democratic Control finanzierten Zeitschrift "Foreign Affairs» auf die Situation der Flüchtlinge aufmerksam und wies auf die Dringlichkeit finanzieller Hilfe hin ${ }^{201}$ - genauso wie Charles Hobhouse im "Manchester Guardian ${ }^{202}$.

Am 25. November 1925 ging der IKRK-Delegierte Dr. Henri Mentha in Tanger an Land. Er sollte sich über die Situation der Flüchtlinge informieren $^{203}$. Mentha traf sich mit dem französischen und dem spanischen Generalkonsul, suchte die Krankenhäuser der Stadt auf und diskutierte mit Pfarrer Elson, Lady Drummond Hay, Feridah Kirby Green und mit dem aus London gekommenen Vertreter der British Red Crescent Society C.W. Kendall über die Lage. Ein für den 3. Dezember 1925 geplantes Treffen mit Generalresident Théodore Steeg in Rabat kam nicht zustande, da dieser seine Abreise nach Frankreich vorbereitete. Drei Tage später kehrte Mentha mit der Erkenntnis nach Genf zurück, daß das IKRK in Tanger nicht zu intervenieren brauche. $\mathrm{Ob}$ neben der Arbeit Elsons weitere Hilfe nötig sei, müßten Frankreich und Spanien allein entscheiden ${ }^{204}$.

${ }^{197}$ Who Was Who 4 (1952) S. 547; Michael STENTON, Stephen LEES, Who's Who of British Parliament. A Biographical Dictionary of the House of Commons, Bd. 2: 1886-1918, London 1978 , S. 175.

${ }^{198}$ Who Was Who 6 (1972) S. 405; PRO-FO 371, 9866, 13373, 132: Embassy of Berlin an FO, 3.10.1924. Frys Autobiographie geht nicht auf die marokkanischen Erlebnisse ein [Anna R. FRY, Ein Quäker-Wagnis. Die abenteuerliche Geschichte eines Friedensfeldzuges in und nach dem Weltkriege, Nürnberg 1933].

${ }_{199}$ PRO-FO 371, 11075, 18, 71: CG Tangier an FO, 7.8.1925.

${ }^{200}$ Refugees in Tangier, in: Al-Moghreb al Aksa \& Tangier Gazette, 8.8.1925.

${ }^{201}$ FRY, Tangier, S. 69.

${ }^{202}$ HOBHOUSE, Relieving Refugees, 1.9.1925. Die untersuchten Quellen geben aber keine Auskunft darüber, ob die Society of Friends tatsächlich Geldspenden nach Tanger geschickt hat.

${ }^{203}$ DuRAND, Histoire, Bd. 2, S. 199; La Croix Rouge et le Rif, in: BISCR 56 (1925) S. 946; CADN Tanger C37: MAE an CG Tanger, 26.11.1925.

${ }^{204}$ MENTHA, Mission, S. 971-973, S. 981. 
So stand der Refugee Relief Fund im April 1926 vor dem finanziellen Aus. Seit März 1924 waren 274847 Francs für Hilfsgüter ausgegeben worden. Jetzt waren die Helfer nicht mehr in der Lage, mehr als ein Kilo Mehl pro Woche an Bedürftige abzugeben. In einem Brief an die "Al-Moghreb Al Aksa \& Tangier Gazette« baten Lady Alice Drummond Hay und H.P. Elson um neue Spenden ${ }^{205}$. Die Notwendigkeit für Hilfsmaßnahmen änderte sich auch nach dem Ende des Rifkrieges nicht. Als der amerikanische Ethnologe Carleton Coon und seine Ehefrau sich im Juni 1926 in Tanger aufhielten, war die Stadt noch voller Flüchtlinge ${ }^{206}$. Ab November 1926 entspannte sich die Situation etwas. Langsam kehrten die Flüchtlinge in ihre Heimat zurück, auch wenn dort die Armut groß war $^{207}$.

\subsection{Die französischen Hilfsmissionen zu den Kriegsgefangenen}

Seit Anfang 1926 gab es Bemühungen, den im Rif festgehaltenen Kriegsgefangenen medizinische Hilfe zukommen zu lassen. In Begleitung des Rifagenten Si Mohammed Chbibi begab sich der französische Journalist Auguste Montagne von der Zeitung »Tribune marocaine« von Februar bis März 1926 mit ersten Hilfsgütern ins Rif. Am 19. Februar 1926 durfte er das französische Gefangenenlager in Sidi Abdallah ben Youssef besuchen. Als Zeichen des guten Willens wurde ihm von Abdelkrim erlaubt, zwei Schwerverletzte hinter die französischen Linien zu bringen ${ }^{208}$.

Auch Pierre Parent plante Hilfe für die Gefangenen im Rif. Er war der Präsident der Fédération marocaine des mutilés et anciens combattants aus Casablanca, die sich seit dem Kriegseintritt Frankreichs im April 1925 an der Front und in Hospitälern um Verwundete kümmerte. Seit November 1925 bemühte sich Parent über verschiedene Kontaktstränge - darunter den Kontrollbeamten von Taourirt Léon Gabrielli -, Näheres über das Schicksal der französischen und spanischen Gefangenen im Rif zu erfahren. Schließlich gelang es ihm im Februar 1926, über den in Tanger lebenden Inspektor für indigene Angelegenheiten Moisès H. Azancot, mit dem Tangerer Agenten Abdelkrims Moulay Ali

${ }^{205}$ Alice DRUMMOND-HAY, H.P. ELSON, Correspondence (Leserbrief), in: Al-Moghreb al Aksa \& Tangier Gazette, 10.4.1926.

${ }^{206}$ Carleton S. COON, Adventures and Discoveries. The Autobiography of Carleton S. Coon, Englewood Cliffs 1981, S. 35.

${ }^{207}$ PRO-FO 371, 11916, 76, 8: CG Tangier an FO, 25.11.1926 mit beigefügtem Brief H.P. Elson an Miss Drummond Hay, 21.11.1926.

${ }^{208}$ Les prisonniers français au Maroc, in: L'Illustration 84 (1926) S. 334; LE GUILLERME, CH. »402«, S. 170f,; Abd-el-Krim Telis of Hope for Peace, in: New York Times, 25.4.1926; Bosque CoMA, Prisionero, S. 26. 
el Khamlichi in Verbindung zu treten. Parent bot auch Medikamente und Verbandszeug für kranke und verwundete Rifkabylen an. Anfang März 1926 signalisierte Azancot Abdelkrims Zustimmung für ein Treffen. Mit seinen Begleitern Bou Tahar, einem Nachrichtenläufer Abdelkrims aus Tanger, und $\mathrm{Si}$ Mohammed Chbibi - der bereits Auguste Montagne begleitet hatte - überquerte Parent am 29. März 1926 die Grenzen des Rifs. Zwei Tage später traf er in Sidi Abdallah ben Youssef mit Außenminister Si Mohammed Azerkane und mit Abdelkrim zusammen. Letzterer erlaubte Parent, sich im Rif frei zu bewegen. Die Gefangenen befanden sich in einem schlechten Zustand. Im Rif hatte sich die Typhusepidemie, die auch vor den fünf Gefangenenlagern bei Sidi Abdallah ben Youssef, Bou Salah, Bouham, Tamasint und Toufist nicht Halt machte, noch verschlimmert ${ }^{209}$. Sehr wahrscheinlich bekamen die Insassen auch die Folgen der spanischen Giftgaseinsätze zu spüren.

In allen Lagern hörte Pierre Parent Berichte über die aufopferungsvolle Hilfe eines gewissen Caïd Abdeslem bzw. Caïd Toubib. Es handelte sich um einen deutschen Deserteur namens Karl Ohme. Zusammen mit seinem Freund Franz Koberstein war der Feldwebel der französischen Fremdenlegion am 28. Mai 1925 bei Taounat im Sektor Taza in die Hände der Rifkabylen gefallen $^{210}$. Es ist dabei nicht klar, ob die beiden desertierten oder ob sie gefangengenommen wurden. Als Assistent und Telefonist für Caïd Haddou verwandelte sich Ohme in den einheimische Kleidung tragenden Caïd Abdeslem, der sich zusammen mit Franz Koberstein auch um die Gefangenenlager kümmerte und die Kranken - Gefangene wie Rifkabylen - während der Typhusepidemie versorgte ${ }^{211}$.

Am 5. April 1926 traf Parent nochmals mit Abdelkrim in Tamasint zusammen, um mit ihm die Modalitäten für eine weitere Hilfsmission zu besprechen. Diese wartete bereits in Taourirt unter der Leitung von Dr. Gaud von der Rabater Direction de la santé et de l'hygiène publique auf das Startsignal ${ }^{212}$. Die

${ }^{209}$ PARENT, Au Riff, S. 26, S. 31-47, S. 50-53, S. 303-307, S. 313, S. 316-329; DUMAS, Abd-el-Krim, S. 124f.; ABDELKRIM, Memoiren, S. 113; Martin GILBERT, Geschichte des 20. Jahrhunderts, Bd. 2: 1919-1933, München 1998, S. 202; Das Problem von Marokko, in: Neue Zürcher Zeitung, 8.4.1926; Les prisonniers français au Maroc, in: L'Illustration 84 (1926) S. 334; Die Lage in Marokko, in: Neue Zürcher Zeitung, 9.4.1926; Le typhus décimerait les Rifains? Abd el Krim demanderait aide sanitaire, in: Petit Journal, 9.4.1926.

${ }^{210}$ Les allemands Ohme, ex-caïd Abdeslem, et Koberstein, poursuivi pour désertion, sont condamnés à vingt ans de travaux forcés, in: Écho du Maroc, 4.2.1927; PA-AA R70857: Antoine Paoli an Botschaft Paris, 9.7.1926.

211 SHAT 3H442: Note de renseignements o.D. [Mitte 1926]; ADMAE Maroc 138, 133: Bericht »Déclarations du caporal Gomis Julio«, 8.6.1926; Le dernier crime des conseils de guerre. Vingts ans de travaux forcés à deux légionnaires faits prisonniers à Maroc, in: Populaire, 22.7.1927. Siehe zu Ohme und Koberstein auch Kapitel 4, S. 122, S. 126, S. 131, S. 149f. und Kapitel 8, S. 371-373.

${ }^{212}$ PARENT, Au Riff, S. 558-562, S. 568-570; CADN Maroc DAI 199: Bericht »Rapport du docteur Gaud, Mission médicale du Riff, mai 1926« o.D. 
Friedenskonferenz von Oujda vom 18. April bis zum 1. Mai 1926 verzögerte den Abmarsch. Spanien und Frankreich verlangten unter anderem die sofortige Freilassung aller Gefangenen. Daran sçheiterte die Konferenz letztendlich, denn Abdelkrim wollte das letzte Faustpfand, das er zu dieser Zeit noch hatte, auf keinen Fall aufgeben ${ }^{213}$.

Mit Frankreich wollte es sich Abdelkrim allerdings nicht vollends verderben. Daher erlaubte er die Entsendung einer rein französischen Hilfsdelegation. Daß diese Geste in Frankreich ein positives Echo hervorrufen würde, war $\mathrm{ihm}$ sicher bewußt. Nicht von ungefähr stellte er sich bei seiner Kapitulation am 27. Mai 1926 den Franzosen und nicht den Spaniern. Als am 12. Mai 1926 das französische Torpedoboot "Sénégalais" mit Hilfsgütern bei Bou Sekour landete, durften nur Pierre Parent, Dr. Gaud und der Krankenpfleger Abdeslem an Land. Ein spanischer Arzt, der in Melilla zugestiegen war, mußte an Bord bleiben. Prinzipiell wurde den Rifsoldaten untersagt, sich dem Konvoi zu nähern. Caìd Haddou verbot den Ärzten in Sidi Abdallah ben Youssef, Einheimischen ohne Erlaubnis der Rifregierung ärztlich beizustehen. $\mathrm{Zu}$ diesem Zeitpunkt wollte Abdelkrim noch Fraternisierungen vermeiden ${ }^{214}$.

Vom Konferenzort Oujda aus hatte sich derweil am 6. Mai 1926 eine zweite französische Mission mit den heimkehrenden Delegierten Abdelkrims ins Rif aufgemacht. Dr. Mosnier, ein Arzt aus Oujda, wurde vom Krankenpfleger Croza und von Dr. Valeton nach Toufist begleitet. Als sich der militärische Zusammenbruch der Riftruppen abzeichnete, wurde die Lage der Gefangenen immer gefährlicher. Aufgebrachte Rifkabylen drohten, sie zu töten. Karl Ohme ließ an die Ärzte zu ihrer Verteidigung Gewehre und Munition verteilen ${ }^{215}$. Am 26. Mai 1926 - einen Tag vor seiner Kapitulation - ließ Abdelkrim die 105 spanischen, 76 französischen und 112 algerisch-senegalesischen Gefangenen aufgrund der eindringlichen Bitten von Dr. Gaud und Dr. Mosnier frei ${ }^{216}$.

${ }^{213}$ PENNELL, A Country, S. 212f.; PRO-FO 371, 11908, 5, 109: Consul General of Rabat an FO, 10.5.1926.

${ }^{214}$ CADN Madrid C261: MAE an Ambassade de Madrid, 8.5.1926; PARENT, Au Riff, S. 572-586; ABDELKRIM, Memoiren, S. 119; GODED LLOPIS, Marruecos, S. 90.

${ }_{215}$ Après l'échec de la conférence d'Oujda, in: Écho de Paris, 7.5.1926; La mission sanitaire, in: L'Illustration 84 (1926) S. 594; PARENT, Au Riff, S. 573; DUMAS, Abd-el-Krim, S. 125f.; SHAT 3H442: Bericht „Renseignements sur les trois légionnaires suspects« o.D. [Mitte 1926]; CADN Maroc DAI 199: Bericht »Rapport du docteur Gaud«.

${ }^{216}$ CADN Madrid C261: MAE an Ambassade de Madrid, 26.5.1926; PARENT, Au Riff, S. 106; Henry CLERISSE, La soumission du Rif, in: L'Illustration 84 (1926) S. $594 f$. 